\title{
MetaGater: Fast Learning of Conditional Channel Gated Networks via Federated Meta-Learning
}

\author{
Sen Lin ${ }^{1}$, Li Yang ${ }^{1}$, Zhezhi He ${ }^{2}$, Deliang Fan $^{1}$, Junshan Zhang ${ }^{1}$ \\ ${ }^{1}$ School of ECEE, Arizona State University \\ ${ }^{2}$ Department of Computer Science and Engineering, Shanghai Jiao Tong University \\ \{slin70, lyang166, dfan, junshan.zhang\}@asu.edu, zhezhi.he@sjtu.edu.cn
}

\begin{abstract}
While deep learning has achieved phenomenal successes in many AI applications, its enormous model size and intensive computation requirements pose a formidable challenge to the deployment in resource-limited nodes. There has recently been an increasing interest in computationallyefficient learning methods, e.g., quantization, pruning and channel gating. However, most existing techniques cannot adapt to different tasks quickly. In this work, we advocate a holistic approach to jointly train the backbone network and the channel gating which enables dynamical selection of a subset of filters for more efficient local computation given the data input. Particularly, we develop a federated metalearning approach to jointly learn good meta-initializations for both backbone networks and gating modules, by making use of the model similarity across learning tasks on different nodes. In this way, the learnt meta-gating module effectively captures the important filters of a good meta-backbone network, based on which a task-specific conditional channel gated network can be quickly adapted, i.e., through one-step gradient descent, from the meta-initializations in a two-stage procedure using new samples of that task. The convergence of the proposed federated meta-learning algorithm is established under mild conditions. Experimental results corroborate the effectiveness of our method in comparison to related work.
\end{abstract}

\section{Introduction}

The last decade has witnessed an explosive boost in deep learning, especially Deep Neural Networks (DNN), leading to phenomenal successes in many artificial intelligence applications, e.g., speech recognition [36], image classification [23, 51], object detection [57, 43] and etc. Nevertheless, DNNs nowadays have very complex structures, e.g., a larger model depth and width for a more expressive representation power, which is not amendable to the de-
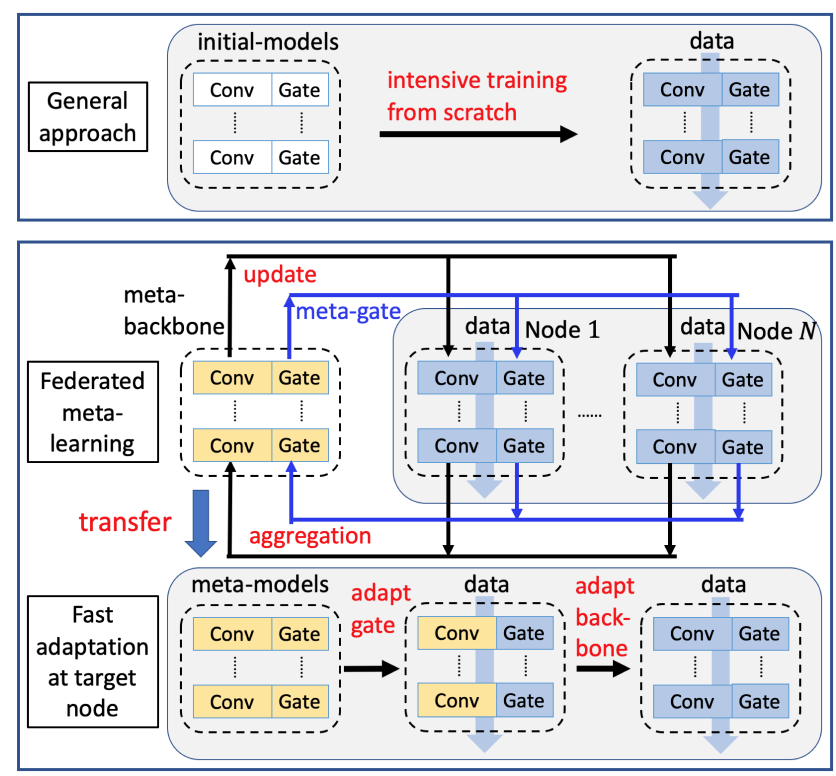

Figure 1. Illustration of the proposed framework MetaGater. Top: general learning of conditional channel gated networks. Bottom: fast learning of conditional channel gated networks via joint federated meta-learning of backbone and gating module.

ployment in resource-constrained settings (e.g., edge servers or robots [42]). This challenge has spurred significant effort on computationally-efficient learning methods recently, including weight quantization [21, 27, 24], weight pruning [22, 62, 63] and channel gating [60, 10, 1]. Notably, both weight pruning and channel gating aim to effectively select only a portion of model parameters, i.e., a sub-network, for local computation (inference) with minimal performance loss, through a sampling mask on either the weights directly, or the channels.

However, most aforementioned studies are afflicted with some of the following limitations: 1) Extensive training cost or poor performance. Generally, the learning of an efficient subnet for a resource-limited node requires substantial pre-training on a large target dataset, which often takes place in a powerful cloud datacenter [63]. Such a learning 
strategy, however, may not be practical due to the concerns on cost and privacy, as a significant amount of data need to be transmitted from the node to the server. It is worth noting that some recent works $[38,50]$ propose to quickly prune a randomly initialized DNN, and then fine-tune the subnet for eliminating the need of pre-training, which however suffers from poor performance and may not actually speed up the inference, as a drawback for unstructured pruning [62]. (2) Limited adaptability to different tasks. The fact that different nodes usually have different local data distributions [46, 32, 53], implies that a global background model is not sufficient to guarantee universally satisfactory learning performance across different tasks. Accordingly, the subnet selection should also vary for different tasks. Yet, most works $[45,1]$ consider a common background model and require non-trivial re-training of masks with massive training data when applied to different tasks. In a nutshell, for effective learning at resource-limited nodes (devices), it is desirable for the learning of subnets for each new task to be able to quickly adapt while incurring minimum training cost, akin to cognitive learning by human beings.

To tackle these challenges, we propose MetaGater, a fast learning framework for conditional channel gated networks by leveraging the knowledge from many nodes, where the backbone network model at each node is associated with a task-specific channel gating module. The channel gating module can generate a data-dependent mask, i.e., a binary vector, for each layer in the backbone network, which dynamically selects a subset of filters to participate into the computation conditioned on the data input, thereby improving the computation efficiency. Since the learning tasks across different nodes often share some similarity [4, 55, 2], we advocate a federated meta-learning approach to jointly learn good meta-initialization for both the backbone networks and the channel gating modules, via the collaboration of many nodes in a distributed manner. The learnt meta-backbone network and the meta-gating module are then transferred from the cloud to a target node for fast adaptation (as shown in Fig. 1). Since the meta-gating module effectively captures the important filters of a good meta-backbone network and hence sparsity structure across tasks, it can achieve the agile adaptability at different new tasks by quickly learning a task-specific conditional channel gated network using corresponding new data samples.

The main contributions of this work can be summarized as follows.

(1) To achieve fast and adaptive learning of subnets on resource-limited nodes, we propose MetaGater, a fast learning framework of conditional channel gated networks via federated meta-learning, where good meta-initializations for both backbone networks and gating modules are jointly learnt by leveraging knowledge from related tasks. A taskspecific conditional channel gated network for a new task can then be learned quickly from the meta-initialization in a two-stage procedure, using data samples of the new task.

(2) To efficiently solve the federated meta-learning problem with non-smooth objective functions, we develop a novel approach based on accelerated proximal gradient descent with inexact solutions to the local problems. Particularly, we use accelerated gradient descent for the meta-backbone network and accelerated proximal gradient descent for the meta-gating module, in the same spirit with the Nesterov's method [47]. By characterizing and controlling the estimation error introduced by the inexact solutions, we establish the convergence of the proposed federated meta-learning algorithm for non-convex functions under mild conditions, and show that an $\epsilon$-first order stationary point can be obtained in at most $O\left(\epsilon^{-1}\right)$ communication rounds.

(3) We conduct experiments to evaluate the effectiveness of MetaGater. Specifically, the experiments on various datasets showcase that the proposed federated meta-learning approach clearly outperforms existing baselines in terms of accuracy and efficiency. Since this study focuses on the fast learning performance based on distributed learning, most existing methods based on centralized pre-training on a large target dataset cannot directly serve as the baseline. For a fair comparison, we develop a new baseline MetaSNIP by integrating federated meta-learning with one state-of-the-art fast pruning approach SNIP [38], where we apply SNIP to the meta-backbone network obtained by using the federated meta-learning approach. Our experimental results indicate that MetaGater is able to quickly obtain a task-specific subnet with higher accuracy, and achieves a larger diversity in the task-model sparsity after fast adaptation, compared with MetaSNIP. This implies that MetaGater can successfully find the joint model of the meta-backbone network and meta-gating module that is sensitive to changes in the tasks, such that quick adaptation in the model parameters will lead to a good task-specific channel gated network for efficient inference.

\section{Related Work}

Federated meta-learning. Meta-learning is a promising solution for fast learning, where one gradient-based metalearning algorithm called MAML [16] has become a representative method. The idea of MAML is to learn a model initialization based on many related tasks, such that even one-step gradient descent from this initialization can achieve good performance for a new task using a few data samples from that task. A lot of work have been proposed to understand [3, 13, 30] and improve upon MAML [40, 48, 65].

Recently, the marriage of federated learning and metalearning has garnered a lot of attention, giving rise to a new research direction, namely federated meta-learning. In particular, the empirical successes of such an integration have been corroborated in $[9,31]$. From a theoretic point of view, 
the work [41] establishes the convergence of federated metalearning for strongly convex functions and investigates the impact of task similarity. Besides, [14] studies the case of non-convex functions with stochastic gradient descent. All the works above have studied federated meta-learning based on MAML-type methods. Recently, a different federated meta-learning approach is proposed in [12], based on a proximal meta-learning method with moreau envelopes. However, to our best knowledge, we are the first to study the agile adaptability and computational efficiency when federated meta-learning is leveraged to jointly learn the backbone network and the gating module, and further analyze the convergence performance for non-smooth functions in this setting. More importantly, the federated meta-learning approach proposed in this work clearly outperforms the previous studies as shown in the experiments.

Channel gating and weight pruning. The idea of utilizing data-dependent channel gating module [7, 54, 26, 17, 25] to improve the computational efficiency has recently emerged in the literature. Specifically, [10] proposes Gaternet to train a separate gating network to select filters for each layer in the backbone network. To increase the amount of conditional features actually learned, a batch-shaping technique is introduced in [6] to learn conditional channel gated networks. [1] further applies the channel gating module to address the catastrophic forgetting in task-aware continual learning, by predicting the current task in a set of pre-defined tasks. The works in network pruning can be traced back to early 1990s [52], where sparsity enforcing penalty terms (e.g., $\mathcal{L}_{0}$ and $\mathcal{L}_{1}$ norm) [61,28] and saliency criterions like weight sensitivity [33] are widely used. Recently, using magnitude of weights [21] as the criterion has achieved significant successes and become a standard method for network pruning, which however needs expensive pruneretrain cycles. SNIP [38] proposes a fast pruning method based on connection sensitivity without pre-training. [50] directly finds optimal subnets in randomly weighted neural networks. Nevertheless, it is difficult for these unstructured pruning methods to reduce the inference time on hardware due to the highly irregular sparsity patterns. Therefore, many approaches in structured pruning $[62,39,44]$ have been proposed to prune weights grouped in regular shapes, such as channels or kennels.

Note that an independent and concurrent work [56] also proposes to utilize meta-learning for rapid structural pruning of neural networks. We highlight the main differences below: 1) [56] relies on a centralized meta-learning method where the nodes are required to submit data to a central platform, whereas we consider a more realistic distributed setup and propose a new federated meta-learning approach to fit the specific efficiency problem in our work. 2) [56] takes a stochastic approach and learns a task-specific Bernoulli
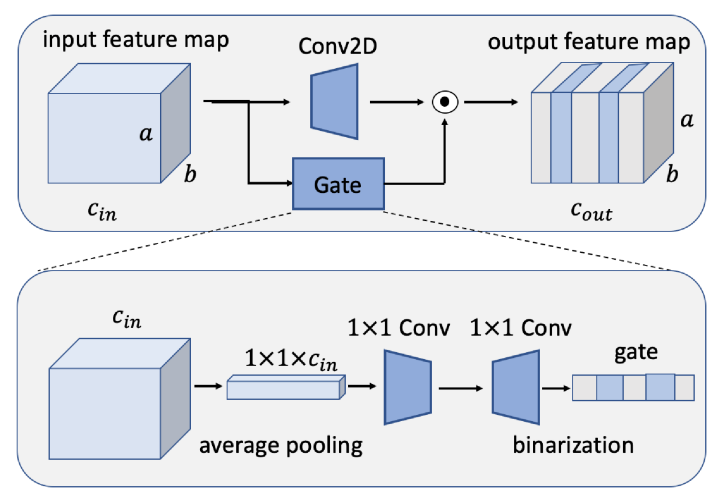

Figure 2. The channel gating module for a convolution layer.

distribution for mask generation, which however could possibly generate masks that lead to significant performance degradation. In stark contrast, we develop a deterministic approach by learning a task-specific channel gating module, and also provide theoretic foundations by carrying out a thorough convergence analysis of the proposed federated meta-learning algorithm.

\section{Methodology}

In this section, we first present the problem formulation for learning task-specific conditional channel gated networks, and then introduce the proposed federated meta-learning for conditional channel gated networks through the collaboration among a set of nodes, followed by the fast adaptation procedure at a target node.

\subsection{Problem formulation}

As alluded to earlier, we integrate a task-specific channel gating module with the backbone network at each node, so as to improve the computational efficiency with a datadependent channel sampling mask.

Backbone network. For a node $i$, let $\tilde{\theta}^{i}$ denote the model parameters for the backbone neural network with $J$ convolutional layers, which serves as the main model that extracts features from the data input and makes predictions.

Channel gating module. Let $\tilde{\phi}^{i}$ denote the model parameters for the channel gating module $Q_{i}=\left[Q_{i}^{1}, \ldots, Q_{i}^{J}\right]$ at node $i$. As depicted in Fig. 2, let $o^{j} \in \mathbb{R}_{\text {in }}^{c^{j}, a, b}$ and $o^{j+1} \in \mathbb{R}^{c_{\text {out }}^{j}, a, b}$ be the input and output feature maps of the $j$-th convolutional layer in the backbone network, respectively. Conditioned on the input feature map $o^{j}$, the layer-wise channel gating module $Q_{i}^{j}$ generates a channel mask vector with binary elements, to determine which channels should be activated for the given input. As a result, a sparse feature map $\hat{o}^{j+1}$, instead of $o^{j+1}$, is forwarded to the next layer, only with the channels activated by the gating module $Q_{i}^{j}$, i.e.,

$$
\hat{o}^{j+1}=Q_{i}^{j}\left(o^{j}\right) \odot o^{j+1}
$$


where $Q_{i}^{j}\left(o^{j}\right)=\left[q_{1}^{j}, \ldots, q_{c_{\text {out }}^{j}}^{j}\right], q_{i}^{j} \in\{0,1\}$ and $\odot$ represents the channel-wise multiplication. Each gating module consists of Multi-Layer Perception (MLP) with a single hidden layer featuring 16 units, followed by a ReLU activation function. To generate the binary mask, we utilize the binarization function [11], and estimate the gradient via straight-through estimator (STE) [8] for the forward and backward paths, respectively. More details about the channel gating module are described in the appendix.

Learning of task-specific channel gated networks. For a target node 0 , let $L_{0}\left(\tilde{\phi}^{0}, \tilde{\theta}^{0}\right)$ denote the empirical loss over the local dataset $D_{0}=\left\{\left(\mathbf{x}_{k}^{0}, \mathbf{y}_{k}^{0}\right)\right\}_{k=1}^{K}$ :

$$
L_{0}\left(\tilde{\phi}^{0}, \tilde{\theta}^{0}\right) \triangleq \frac{1}{\left|D_{0}\right|} \sum_{\left(\mathbf{x}_{k}^{0}, \mathbf{y}_{k}^{0}\right) \in D_{0}} l\left(\tilde{\phi}^{0}, \tilde{\theta}^{0} ;\left(\mathbf{x}_{k}^{0}, \mathbf{y}_{k}^{0}\right)\right)
$$

for some standard loss $l$, e.g., cross-entropy loss. Then, the joint learning of the backbone network and the channel gating module can be formulated as the following regularized optimization problem:

$$
\min _{\tilde{\phi}^{0}, \tilde{\theta}^{0}} L_{0}\left(\tilde{\phi}^{0}, \tilde{\theta}^{0}\right)+\frac{\lambda}{2}\left\|\tilde{\phi}^{0}-\phi\right\|_{2}^{2}+\frac{\lambda}{2}\left\|\tilde{\theta}^{0}-\theta\right\|_{2}^{2}
$$

where $\lambda$ is some constant penalty parameter, $\phi$ and $\theta$ are some prior model parameters for the gating module and the backbone network, respectively. Clearly, directly solving (3), i.e., searching for the optimal task-specific conditional channel gated network, is computationally challenging in general, and possibly suffers from poor performance if only a small local dataset is used for training. On the other hand, the quality of the regularizer plays an important role in controlling the performance of learnt conditional channel gated network, in the sense that the closer the prior parameters are to the task-specific optimal parameters, the better the learning performance is. This regularized learning problem is also intimate with biased regularized hypothesis transfer learning [65], which has thoroughly demonstrated its efficiency in many applications $[15,59,35]$. Therefore, instead of directly solving (3) as in prior works [10, 1], we take a different approach by learning a more informative prior regularizer, such that a quick adaptation at the target node through gradient descent can lead to a good approximation of the optimal subnet.

\subsection{Joint learning of meta-backbone network and meta-gating module via federated meta- learning}

Since the learning tasks across different nodes usually share some similarity, to obtain a good prior regularizer for (3), we develop a new federated meta-learning approach, not only to learn a meta-backbone network but also to learn a meta-gating module, by leveraging the knowledge from related tasks on a set of nodes.

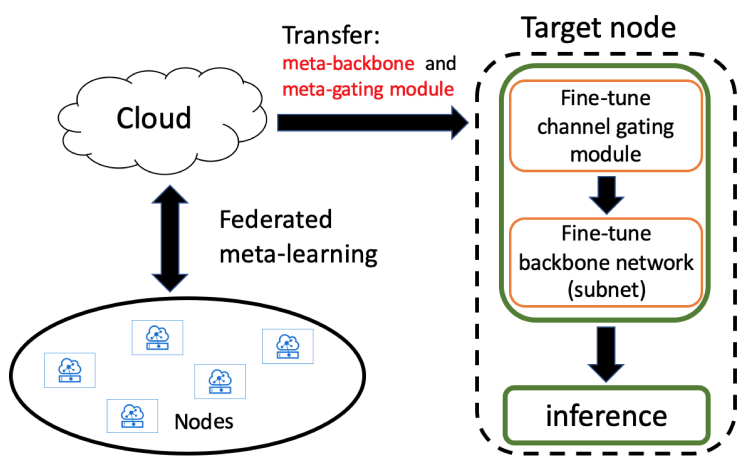

Figure 3. A fast learning framework of conditional channel gated networks via federated meta-learning.

As illustrated in Fig. 3, a set $\mathcal{S}$ of nodes, each $i \in \mathcal{S}$ with a task and a local dataset $D_{i}$, participate into federated meta-learning to jointly learn meta-models for both backbone networks and channel gating modules, which are then transferred via the cloud to a target node for fast adaptation. Let $|\mathcal{S}|=N$. Intuitively, a good meta-model $(\phi, \theta)$ should have the following properties:

- For any $i \in \mathcal{S}$, the meta-model should be 'close' to its task-specific optimal backbone network and gating module, such that the loss is minimized when solving a similar local problem as (3). In this way, the learnt metamodel implicitly captures the way to quickly learn the optimal task-specific conditional channel gated network with local data across all nodes in $\mathcal{S}$.

- In general, a quick adaptation through gradient descent is not sufficient to prompt the sparsity of the task-specific gating module. Instead, it is more effective to start with a meta-gating module with structured sparsity (which also serves as the initialization of fast adaptation), for better computational efficiency.

Therefore, the objective of federated meta-learning can be mathematically formulated as follows:

$$
\begin{gathered}
\min _{\phi, \theta} F(\phi, \theta)=H(\phi)+\frac{1}{N} \sum_{i=1}^{N} \min _{\tilde{\phi}^{i}, \tilde{\theta}^{i}} G_{i}\left(\tilde{\phi}^{i}, \tilde{\theta}^{i}\right) \\
\text { s.t. } G_{i}\left(\tilde{\phi}^{i}, \tilde{\theta}^{i} ; \phi, \theta\right)=L_{i}\left(\tilde{\phi}^{i}, \tilde{\theta}^{i}\right)+\frac{\lambda}{2}\left\|\tilde{\phi}^{i}-\phi\right\|_{2}^{2}+\frac{\lambda}{2}\left\|\tilde{\theta}^{i}-\theta\right\|_{2}^{2},
\end{gathered}
$$

where $L_{i}\left(\tilde{\phi}^{i}, \tilde{\theta}^{i}\right)$ is the empirical loss defined in a similar way with (2) for node $i$, and $H(\cdot)$ is some sparsity prompting function of the meta-gating module, such as $\mathcal{L}_{1}$-norm and Group Lasso [64]. It is worth noting that compared with the popular gradient-based meta-learning method MAML [16], such a regularization-based meta-learning formulation can fully leverage the higher-order information [65] of the objective function (4), leading to more informative meta-backbone networks and meta-gating modules.

The next key question is how to efficiently solve problem (4) in a distributed manner. To answer this question, 
there are some problems to be solved: 1) Computationallyefficient methods usually result in performance degradation, compared with that of the entire backbone network. To guarantee the performance of conditional channel-gated networks after fast adaptation, a better meta-backbone network is needed, given a fixed communication budget (between the cloud and nodes) which is often a significant bottleneck in wireless networks. 2) Generally $H(\cdot)$ is a non-smooth function such that the classical gradient descent may not work well.

To address the above problems, we develop a new federated meta-learning approach based on accelerated proximal gradient descent [19], as summarized in Algorithm 1. In what follows, we highlight several key aspects in our algorithm design.

- Generally, it is computationally expensive to find a local minimizer to problem (5) (in Algorithm 1) at each node. Instead, we run the vanilla gradient descent for several steps to approximately solve (5), for the case when $G_{i}$ is smooth for a smooth local loss $L_{i}$. In this way, Algorithm 1 would obtain a meta-model $(\phi, \theta)$ such that the conditional channel gated network, obtained after fast adaptation via several gradient descent steps at each node, can achieve good learning performance.

- Unlike the meta-model update in MAML which includes the computation of Hessian, the global update of meta-models in Algorithm 1 is as easy to implement as first-order meta-learning algorithms, e.g., Reptile [48]. Particularly, let $\left(\tilde{\phi}_{t}^{i *}, \tilde{\theta}_{t}^{i *}\right)=$ $\arg \min _{\left(\tilde{\phi}^{i}, \tilde{\theta}^{i}\right)} G_{i}\left(\tilde{\phi}_{t}^{i}, \tilde{\theta}_{t}^{i} ; \phi_{t}^{p r}, \theta_{t}^{p r}\right)$. For the $t$-th iterate $\left(\phi_{t}^{p r}, \theta_{t}^{p r}\right)$ of the meta-model in Algorithm 1, it can be shown $[65,58]$ that $\left(\tilde{\phi}_{t}^{i *}, \tilde{\theta}_{t}^{i *}\right)$ satisfies:

$$
\begin{aligned}
\lambda\left(\phi_{t}^{p r}-\tilde{\phi}_{t}^{i *}\right) & =\nabla_{\phi_{t}^{p r}} G_{i}\left(\tilde{\phi}_{t}^{i *}, \tilde{\theta}_{t}^{i *} ; \phi_{t}^{p r}, \theta_{t}^{p r}\right), \\
\lambda\left(\theta_{t}^{p r}-\tilde{\theta}_{t}^{i *}\right) & =\nabla_{\theta_{t}^{p r}} G_{i}\left(\tilde{\phi}_{t}^{i *}, \tilde{\theta}_{t}^{* *} ; \phi_{t}^{p r}, \theta_{t}^{p r}\right),
\end{aligned}
$$

which indicate that the global updates of meta-model (step 8 and 9 in Algorithm 1) follow an approximate gradient direction with respect to (w.r.t.) the meta-learning objective (4).

- Since the meta-learning objective $F$ is a non-smooth function w.r.t. $\phi$, we apply proximal gradient descent for the global update of the meta-gating module. More specifically, the proximal operator [49] of function $H$ is defined by

$$
\operatorname{prox}_{\eta H}(v)=\arg \min _{x}\left(H(x)+\frac{1}{2 \eta}\|x-v\|_{2}^{2}\right)
$$

for $\eta>0$. When $H=0$, it is clear that $\operatorname{prox}_{\eta H}(v)=v$. Hence, if $v$ is a standard gradient step as in step 9 of Algorithm 1, $\operatorname{prox}_{\eta H}(v)$ can be interpreted as trading off minimizing $H$ and being close to $v$.

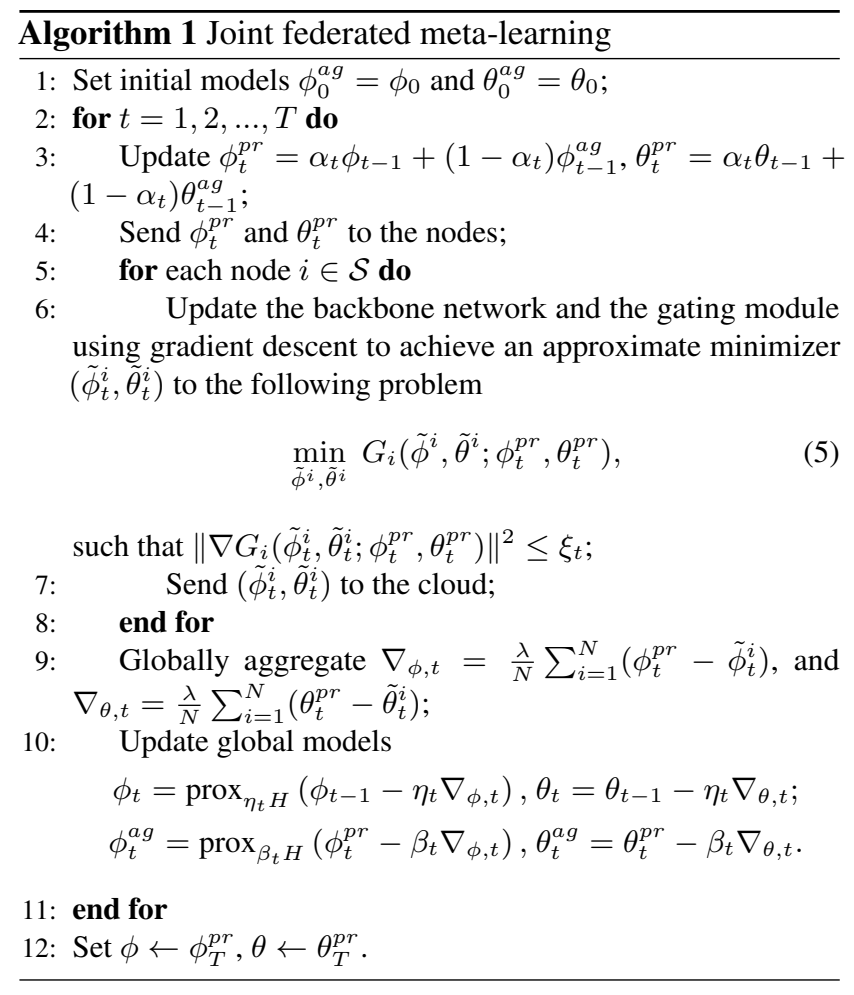

- Since the global updates of meta-models indeed follow an approximate gradient direction w.r.t (4) (gradient descent for meta-backbone network and proximal gradient descent for meta-gating module), we resort to a general acceleration technique [19] for the global updates to improve the performance of federated meta-learning. When $\beta_{t}=\eta_{t} \alpha_{t}$, the global updates of meta-models fall into the variants of the well-known Nesterov's method [47].

\subsection{Fast adaptation at the target node}

Based on the meta-backbone network $\phi$ and the metagating module $\theta$ transferred from the cloud, the target node 0 is able to quickly learn a task-specific conditional channel gated network. Different from the simultaneous updates of both backbone networks and gating modules in federated meta-learning, the gating module and the backbone network are updated once sequentially, at the target node by following a two-stage procedure using its local dataset $D_{0}$ :

Stage I: Fix the task-specific backbone network as the meta-backbone network $\theta$, and update the task-specific gating module via one-step gradient descent from the metagating module $\phi$ w.r.t. (3) using dataset $D_{0}$. Note that $\phi$ has effectively captured the important filters of a good metabackbone network by leveraging the knowledge among different nodes in $\mathcal{S}$. Therefore, one single gradient update from $\phi$ by incorporating the local information is able to tune the gating module in a way that important channels for 
the specific task can be quickly selected, thus significantly reducing the network size for updating.

Stage II: Given the adapted channel gating module $\tilde{\phi}^{0}$, which determines the set of filters for local computation, we next fine-tune the subnet via one-step gradient descent from the corresponding subnet in the meta-backbone model $\theta$, using the local dataset $D_{0}$. In this way, the training cost is further reduced as only a part of the backbone network gets involved in the single forward pass, even more efficient than fast-pruning methods, such as SNIP where at least one single forward pass needs to perform on the entire backbone network.

Therefore, a task-specific conditional channel gated network can be quickly obtained at the target node for efficient inference.

\section{Theoretical Analysis}

In this section, we present the convergence analysis of Algorithm 1 for a general non-convex local loss function.

First, a key observation here is that from the perspective of convergence analysis, the updates of the meta-backbone networks, i.e., vanilla gradient descent, in step 9 of Algorithm 1 are equivalent to the following proximal gradient descent:

$$
\begin{aligned}
\theta_{t} & =\operatorname{prox}_{\eta_{t} H}\left(\theta_{t-1}-\eta_{t} \nabla_{\theta, t}\right), \\
\theta_{t}^{a g} & =\operatorname{prox}_{\beta_{t} H}\left(\theta_{t}^{p r}-\beta_{t} \nabla_{\theta, t}\right),
\end{aligned}
$$

because $H$ is a function only of the meta-gating module $\phi$ and $\nabla_{\theta} H(\phi)=0$. Consequently, we can analyze the meta-backbone network $\theta$ and the meta-gating module $\phi$ together, and examine the convergence of Algorithm 1 w.r.t. $w=(\phi, \theta)$. Let $w_{t}^{p r}=\left(\phi_{t}^{p r}, \theta_{t}^{p r}\right), w_{t}^{a g}=\left(\phi_{t}^{a g}, \theta_{t}^{a g}\right)$ and $\tilde{w}_{t}^{i}=\left(\tilde{\phi}_{t}^{i}, \tilde{\theta}_{t}^{i}\right)$. The step 9 in Algorithm 1 is then equivalent to the following:

$$
\begin{aligned}
w_{t} & =\operatorname{prox}_{\eta_{t} H}\left(w_{t-1}-\eta_{t} \nabla_{w, t}\right), \\
w_{t}^{a g} & =\operatorname{prox}_{\beta_{t} H}\left(w_{t}^{p r}-\beta_{t} \nabla_{w, t}\right),
\end{aligned}
$$

where $\nabla_{w, t}=\frac{\lambda}{N} \sum_{i=1}^{N}\left(w_{t}^{p r}-\tilde{w}_{t}^{i}\right)$.

We next characterize the structural properties of the federated meta-learning objective $F(w)$. For ease of exposition, let $G(w)=\frac{1}{N} \sum_{i=1}^{N} \min _{\tilde{w}^{i}} G_{i}\left(\tilde{w}^{i} ; w\right)$. As is standard, we make the following assumptions.

Assumption 1. The loss function $L_{i}$ is twice-differentiable and $\rho$-smooth, i.e., $\left\|\nabla L_{i}(w)-\nabla L_{i}\left(w^{\prime}\right)\right\| \leq \rho\left\|w-w^{\prime}\right\|$.

Assumption 2. $H(\cdot)$ is a proper closed convex function, and $\|w\| \leq M$. This implies that $\|$ prox $_{c H}(w-c g) \| \leq M$ for any $c>0$ and $g$.

It can be shown that Assumption 2 immediately holds for $\mathcal{L}_{1}$-norm and Group Lasso with bounded domain [19]. Following the same line as in [65], we have the following lemma:
Lemma 1. Suppose that Assumption 1 holds. For $\lambda>\rho$, $G(w)$ is $\frac{\lambda \rho}{\lambda+\rho}$-smooth w.r.t. $w$.

Therefore, the federated meta-learning problem (4) can be rewritten as

$$
\min _{w} F(w)=H(w)+G(w)
$$

where $H(\cdot)$ is convex and non-smooth, and $G(\cdot)$ is nonconvex and smooth. We aim to establish the convergence of Algorithm 1 for finding a first-order stationary point of $F(w)$, which is defined as follows.

Definition 1. $w$ is a first-order stationary point of $F$ if $0 \in$ $\partial H(w)+\nabla G(w)$, where $\partial H(\cdot)$ denotes the subdifferential of $H(\cdot)$.

Let $\mathcal{Q}(w, g, c)=\frac{1}{c}\left[w-\operatorname{prox}_{c H}(w-c g)\right]$. When $g=$ $\nabla G(w), \mathcal{Q}(w, g, c)$ is generally called the gradient mapping at $w$ [49]. It is easy to tell that $\mathcal{Q}(w, \nabla G(w), c)=\nabla G(w)$ if $H(w)=0$. Hence, the value of $\mathcal{Q}(w, g, c)$ is often used as a termination criterion for solving non-smooth optimization problem as a surrogate of the subdifferentials, based on the following lemma $[20,5]$ :

Lemma 2. $w$ is a first-order stationary point of $F$ if and only if $\mathcal{Q}(w, g, c)=0$.

It has been shown in [19] that $\|\mathcal{Q}(w, g, c)\|^{2}$ can be used to quantify the gap between $w$ and the first-order stationary point of $F$, in the sense that this optimality gap converges to 0 as the value of $\|\mathcal{Q}(w, g, c)\|^{2}$ vanishes. Therefore, in this work we seek to establish the convergence of Algorithm 1 in terms of an $\epsilon$-first order stationary point, i.e., $\|\mathcal{Q}(w, g, c)\|^{2} \leq \epsilon$.

As mentioned earlier, the global update direction $\nabla_{w, t}$ is indeed an approximate gradient w.r.t. to function $G$. To show the convergence, we first evaluate this gradient estimation gap, which is captured by the following lemma.

Lemma 3. Suppose that Assumption 1 holds. For $\lambda>\rho$, the following equality holds:

$$
\nabla_{w, t}=\nabla_{w} G\left(w_{t}^{p r}\right)+\delta_{t},
$$

where $\left\|\delta_{t}\right\|^{2} \leq \frac{\lambda^{2} \xi_{t}}{(\lambda-\rho)^{2}}$.

For convenience, denote an auxiliary sequence

$$
\Gamma_{t}= \begin{cases}1, & t=1, \\ \left(1-\alpha_{t}\right) \Gamma_{t-1}, & t \geq 2 .\end{cases}
$$

Let $w^{*}$ be the optimal solution to problem (8), i.e., $F\left(w^{*}\right)=$ $\min _{w} F(w)$. We can have the following main theorem about the convergence of Algorithm 1.

Theorem 1. Suppose that Assumptions 1 and 2 hold. For any $t \geq 1$, let $\alpha_{t}=\frac{2}{t+1}, \beta_{t}<\frac{\lambda+\rho}{\lambda \rho}$ and $\eta_{t}$ satisfy:

$$
\alpha_{t} \eta_{t} \leq \beta_{t}, \frac{\alpha_{t}}{\Gamma_{t}}\left(\frac{1}{\eta_{t}}-1\right) \geq \frac{\alpha_{t+1}}{\Gamma_{t+1}}\left(\frac{1}{\eta_{t+1}}-1\right) .
$$


Then, for $\lambda>\rho$, we have

$$
\begin{aligned}
\min _{t \in[1, T]}\left\|\mathcal{Q}\left(w_{t}^{p r}, \nabla_{w} G\left(w_{t}^{p r}\right), \beta_{t}\right)\right\|^{2} \leq \frac{24 \lambda^{3} \rho \sum_{t=1}^{T} \xi_{t}}{T(\lambda+\rho)(\lambda-\rho)^{2}} \\
+\frac{24(\lambda \rho)^{2}\left(\left\|w^{*}\right\|^{2}+2 M\right)}{T(\lambda+\rho)^{2}}+\frac{C\left\|w_{0}-w^{*}\right\|^{2}}{T^{2}(T+1)}
\end{aligned}
$$

where $C=\frac{24 \lambda \rho}{(\lambda+\rho)}\left(\frac{1}{\eta_{1}}-1\right)$.

It can be seen from Theorem 1 that the convergence error consists of three terms, where the first term captures the impact of inexact solutions to each local learning problem (5), and the last two terms quickly converge to 0 in the faster rate of $O\left(\frac{1}{T}\right)$, compared with the rate of $O\left(\frac{1}{\sqrt{T}}\right)$ in [65]. Let $\psi=\frac{24 \lambda^{3} \rho \sum_{t=1}^{T} \xi_{t}}{T(\lambda+\rho)(\lambda-\rho)^{2}}$. Clearly, if the accumulated estimation error $\sum_{t=1}^{T} \xi_{t}=o(T)$ for local learning problem (5), $\psi$ will vanish eventually. Therefore, Algorithm 1 can find an $O(\epsilon+\psi)$-first order stationary point in at most $O\left(\epsilon^{-1}\right)$ communication rounds between the cloud and nodes.

\section{Experiments}

To evaluate the performance of the proposed framework MetaGater, we seek to answer the following questions in this section: (1) What is the performance of the proposed federated meta-learning approach when compared with other federated meta-learning methods? (2) What is the impact of the channel gating modules on the fast adaptation performance at the target node? (3) What is the performance of MetaGater when compared with other fast pruning methods?

\subsection{Experimental setup}

Datasets. In the experiments, we first study the image classification problem as the learning tasks on two widely used datasets, MNIST [37] and CIFAR-10 [34]. In particular, for MNIST, we distribute the data among $N=20$ tasks, such that the number of samples per task is in the range of $[1165,3834]$. We randomly select 5 tasks for each round in federated meta-training, and 5 target tasks for fast adaptation. For CIFAR-10, the data is distributed among $N=50$ tasks where the number of samples per task is in the range of [221, 2792]. Similarly, we randomly select 20 tasks for each round in federated meta-training, and 20 target tasks for fast adaptation. Also, each task only has data samples from two classes for both datasets. (Due to the space limitation, we relegate more experimental results to the appendix.)

Models. For MNIST, we consider a two-layer neural network with a hidden layer of size 100. For CIFAR-10, we consider a four-layer convolutional neural network with three convolutional layers, followed by a fully connected layer. The layer sizes are 32, 64, 128 and 2048, respectively. To minimize the introduced model overhead, we integrate the channel gating module at the third convolutional layer, where Group Lasso is used to prompt sparsity of the channel gating module. It is worth noting that the same channel gating technique can be applied to other layers along the same line.

Parameter setup. As is standard in federated learning [46], we evaluate the performance under a fixed number of communication rounds, with $T=800$ and $T=400$ for MINIST and CIFAR-10, respectively. Other hyperparameters are same for both datasets. For $t$-th round, the learning rate $\alpha_{t}=\frac{2}{t+1}$, and we choose $\beta_{t}=\alpha_{t} \eta_{t}=1$. Besides, $\lambda=0.2$. During federated meta-learning, we run gradient descent for multiple local updates to solve the local minimization problem (5) for each training tasks. For fast adaptation at target tasks, we only run one-step gradient descent to fine-tune both meta-backbone network and metagating module. We evaluate the testing accuracy at the target tasks, and repeat all the experiments for 5 times to obtain the average performance.

\subsection{Meta-models via federated meta-learning}

To answer the first question, we consider two existing baseline algorithms, i.e., the classical federated learning algorithm FedAvg [46] and one state-of-the-art federated meta-learning approach Per-FedAvg [14]. For a fair comparison, we first remove the channel gating module, consider meta-learning of the backbone network, and also update the output of FedAvg with one-step gradient descent as in PerFedAvg for testing at the target task. Note that we do not use pFedMe [58] as a baseline for the following reasons: (1) The performance of the global model therein is worse than Per-FedAvg; (2) although the personalized models can achieve better accuracy, it requires to test all personalized models and pick the best one, which is clearly not suitable for on-device learning.

As illustrated in Fig. 4, MetaGater clearly converges faster than FedAvg and Per-FedAvg, which is important in federated learning as the communication cost usually is a bottleneck in wireless networks. Moreover, we have the following observations based on Table 1: (1) MetaGater achieves the best accuracy performance among all the methods. (2) For learning the meta-backbone network only, MetaGater achieves a much higher testing accuracy with less training time compared with Per-FedAvg, even with only one-step gradient update locally during the training process. Note that the longer training time for Per-FedAvg is because it need to evaluate the local gradient for two times in one local update, in order to approximate the gradient w.r.t. the meta-model. Such a performance improvement firmly corroborates the benefits of utilizing higher-order information of the metaobjective function through proximal updates and accelerating the global meta-model updates with momentum. (3) As expected, it takes longer to jointly train the meta-backbone network and the meta-gating module for MetaGater with 


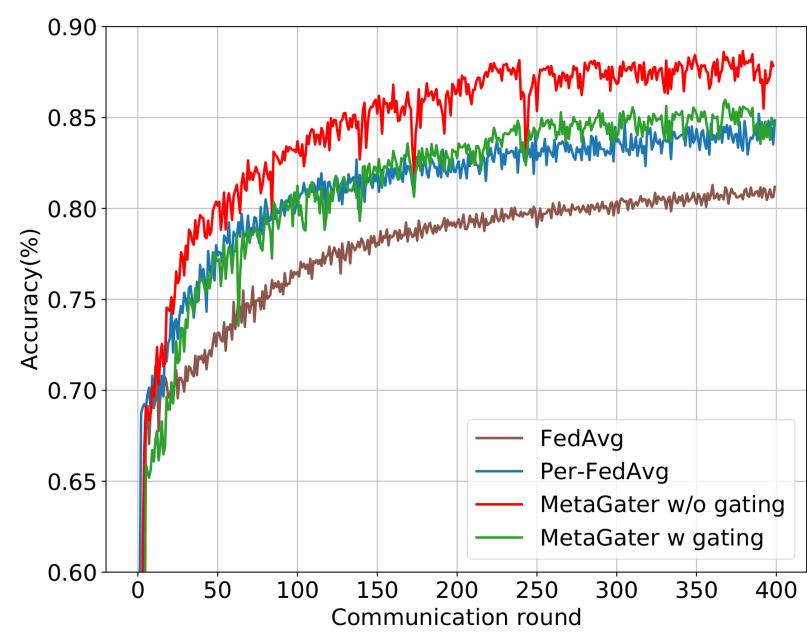

Figure 4. Convergence behavior of MetaGater, Per-FedAvg, FedAvg on CIFAR-10. We compare testing accuracy for target tasks.

\begin{tabular}{c|cccc}
\hline \multirow{2}{*}{ Dataset } & Method & $\begin{array}{c}\text { Local } \\
\text { update }\end{array}$ & Accuracy(\%) & $\begin{array}{c}\text { Training } \\
\text { time(s) }\end{array}$ \\
\hline \multirow{5}{*}{ MNIST } & FedAvg & 1 & $98.78 \pm 0.03$ & 396 \\
& Per-FedAvg & 1 & - & - \\
\cline { 2 - 5 } & MetaGater & 1 & $99.05 \pm 0.05$ & 920 \\
& w/o gating & 2 & $99.4 \pm 0.03$ & 1417 \\
\hline \multirow{5}{*}{ CIFAR10 } & FedAvg & 1 & $80.9 \pm 1.2$ & 1139 \\
& & 2 & - & - \\
\cline { 2 - 5 } & Per-FedAvg & 1 & $82.5 \pm 2.3$ & 2056 \\
\cline { 2 - 5 } & & 2 & - & - \\
\hline & MetaGater & 1 & $88.1 \pm 2.0$ & 1186 \\
& w/o gating & 2 & $88.6 \pm 2.5$ & 2046 \\
\cline { 2 - 5 } & MetaGater & 1 & $87.5 \pm 2.6$ & 1960 \\
& w/ gating & 2 & $88.2 \pm 2.8$ & 3719 \\
\hline
\end{tabular}

Table 1. Accuracy comparison for MetaGater, Per-FedAvg, FedAvg on MNIST and CIFAR-10. Clearly, MetaGater achieves the best accuracy among all methods.

gating, compared to training MetaGater without gating. This extra training time is nevertheless not important from an offline training perspective. More importantly, the structural sparsity offered by the gating module can help to achieve the agile adaptability at different new tasks.

\subsection{Impact of channel gating module}

To highlight the impact of channel gating module, we compare the fast adaptation performance at the target tasks between (1) MetaGater without (w/o) channel gating module and (2) MetaGater with (w/) channel gating module. It can be seen from Table 1 that at the cost of extra training time to jointly meta-train the backbone network and the gating module, MetaGater can achieve the agile adaptability at different target tasks. In particular, for the fast adaptation performance shown in Table 2, with channel gating module, the target task

\begin{tabular}{cccc}
\hline Method & Accuracy $(\%)$ & Sparsity $(\%)$ & Learning time(s) \\
\hline $\begin{array}{l}\text { MetaGater } \\
\text { w/o gating }\end{array}$ & $88.1 \pm 2.0$ & - & 1.2 \\
\hline MetaSNIP & $86.8 \pm 3.3$ & $25 \pm 1.2$ & 1.4 \\
\hline $\begin{array}{c}\text { MetaGater } \\
\text { w/ gating }\end{array}$ & $87.5 \pm 2.6$ & $25 \pm 3.2$ & 1.03 \\
\hline
\end{tabular}

Table 2. Fast adaptation performance comparison on CIFAR-10. The sparsity represents the channel-wise sparsity in the third convolutional layer. Compared with MetaSNIP, MetaGater has a better accuracy and a larger sparsity range. The learning time is the total time for fast adaptation and inference at the target tasks.

is able to quickly obtain a more compact model for efficient inference with only a slightly degradation in the accuracy, compared with MetaGater w/o gating module.

\subsection{Performance of MetaGater}

Since this study focuses on the fast learning performance based on distributed learning, most existing methods based on centralized pre-training on a large target dataset cannot directly serve as a baseline without nontrivial modification. Note that SNIP [38] is a fast pruning method which directly pruns the initial network at single-shot, and hence eliminates the need of pre-training. To fairly evaluate the performance of MetaGater, we compare MetaGater with the following approach (referred as MetaSNIP): we train a metabackbone network using MetaGater without gating module, apply SNIP to quickly obtain a sparse backbone network, and then fine-tune it using one-step gradient descent.

It can be seen from Table 2 that MetaGater clearly outperforms MetaSNIP in the following sense: (1) MetaGater is able to obtain a better subnet with higher accuracy, in a similar speed with MetaSNIP; (2) After fast adaptation, MetaGater exhibits a larger diversity of the achieved model sparsity on different target tasks. This larger diversity implies a better sensitivity of the learnt meta-gating module w.r.t. one-step gradient descent, which enables the fast learning of the task-specific channel gated network.

\section{Conclusion}

In this work, we propose MetaGater, a fast learning framework of conditional channel gated networks via federated meta-learning, where good meta-initializations for both backbone networks and gating modules are jointly learnt by leveraging the model similarity across learning tasks on different nodes. As the meta-gating module effectively captures the important filters of a good meta-backbone network and offers structural sparsity across tasks, it can achieve the agile adaptability at different new tasks by quickly learning a taskspecific conditional channel gated network. Particularly, to efficiently solve the federated meta-learning problem, we 
further propose a novel approach based on accelerated proximal gradient descent with inexact solutions to the local problem, and show that an $\epsilon$-first order stationary point can be obtained in at most $O\left(\epsilon^{-1}\right)$ communication rounds for nonconvex functions. Experiments showcase that our federated meta-learning approach clearly outperforms other existing methods, and MetaGater quickly returns an task-specific channel gated networks after fast adaptation, with a higher accuracy than one state-of-the-art method in fast pruning.

\section{References}

[1] Davide Abati, Jakub Tomczak, Tijmen Blankevoort, Simone Calderara, Rita Cucchiara, and Babak Ehteshami Bejnordi. Conditional channel gated networks for task-aware continual learning. In Proceedings of the IEEE/CVF Conference on Computer Vision and Pattern Recognition, pages 3931-3940, 2020. 1, 2, 3, 4

[2] Alan Aipe and Ujwal Gadiraju. Similarhits: Revealing the role of task similarity in microtask crowdsourcing. In Proceedings of the 29th on Hypertext and Social Media, pages 115-122. 2018. 2

[3] Antreas Antoniou, Harrison Edwards, and Amos Storkey. How to train your maml. arXiv preprint arXiv:1810.09502, 2018. 2

[4] Andreas Argyriou, Theodoros Evgeniou, and Massimiliano Pontil. Convex multi-task feature learning. Machine learning, 73(3):243-272, 2008. 2

[5] Hedy Attouch, Jérôme Bolte, and Benar Fux Svaiter. Convergence of descent methods for semi-algebraic and tame problems: proximal algorithms, forward-backward splitting, and regularized gauss-seidel methods. Mathematical Programming, 137(1-2):91-129, 2013. 6

[6] Babak Ehteshami Bejnordi, Tijmen Blankevoort, and Max Welling. Batch-shaping for learning conditional channel gated networks. In International Conference on Learning Representations, 2019. 3

[7] Yoshua Bengio. Deep learning of representations: Looking forward. In International Conference on Statistical Language and Speech Processing, pages 1-37. Springer, 2013. 3

[8] Yoshua Bengio, Nicholas Léonard, and Aaron Courville. Estimating or propagating gradients through stochastic neurons for conditional computation. arXiv preprint arXiv:1308.3432, 2013. 4, 20

[9] Fei Chen, Mi Luo, Zhenhua Dong, Zhenguo Li, and Xiuqiang He. Federated meta-learning with fast convergence and efficient communication. arXiv preprint arXiv:1802.07876, 2018. 2

[10] Zhourong Chen, Yang Li, Samy Bengio, and Si Si. You look twice: Gaternet for dynamic filter selection in cnns. In Proceedings of the IEEE Conference on Computer Vision and Pattern Recognition, pages 9172-9180, 2019. 1, 3, 4

[11] Matthieu Courbariaux, Itay Hubara, Daniel Soudry, Ran ElYaniv, and Yoshua Bengio. Binarized neural networks: Training deep neural networks with weights and activations constrained to+ 1 or-1. arXiv preprint arXiv:1602.02830, 2016. 4
[12] Canh T Dinh, Nguyen H Tran, and Tuan Dung Nguyen. Personalized federated learning with moreau envelopes. arXiv preprint arXiv:2006.08848, 2020. 3

[13] Alireza Fallah, Aryan Mokhtari, and Asuman Ozdaglar. On the convergence theory of gradient-based model-agnostic meta-learning algorithms. In International Conference on Artificial Intelligence and Statistics, pages 1082-1092, 2020. 2

[14] Alireza Fallah, Aryan Mokhtari, and Asuman Ozdaglar. Personalized federated learning with theoretical guarantees: A model-agnostic meta-learning approach. Advances in Neural Information Processing Systems, 33, 2020. 3, 7

[15] Li Fei-Fei, Rob Fergus, and Pietro Perona. One-shot learning of object categories. IEEE transactions on pattern analysis and machine intelligence, 28(4):594-611, 2006. 4

[16] Chelsea Finn, Pieter Abbeel, and Sergey Levine. Modelagnostic meta-learning for fast adaptation of deep networks. arXiv preprint arXiv:1703.03400, 2017. 2, 4

[17] Xitong Gao, Yiren Zhao, Łukasz Dudziak, Robert Mullins, and Cheng-zhong $\mathrm{Xu}$. Dynamic channel pruning: Feature boosting and suppression. arXiv preprint arXiv:1810.05331, 2018. 3

[18] Saeed Ghadimi and Guanghui Lan. Optimal stochastic approximation algorithms for strongly convex stochastic composite optimization i: A generic algorithmic framework. SIAM Journal on Optimization, 22(4):1469-1492, 2012. 12

[19] Saeed Ghadimi and Guanghui Lan. Accelerated gradient methods for nonconvex nonlinear and stochastic programming. Mathematical Programming, 156(1-2):59-99, 2016. 5, 6,12

[20] Pinghua Gong, Changshui Zhang, Zhaosong Lu, Jianhua Huang, and Jieping Ye. A general iterative shrinkage and thresholding algorithm for non-convex regularized optimization problems. In international conference on machine learning, pages 37-45, 2013. 6

[21] Song Han, Huizi Mao, and William J Dally. Deep compression: Compressing deep neural networks with pruning, trained quantization and huffman coding. arXiv preprint arXiv:1510.00149, 2015. 1, 3

[22] Song Han, Jeff Pool, John Tran, and William Dally. Learning both weights and connections for efficient neural network. In Advances in neural information processing systems, pages 1135-1143, 2015. 1

[23] Kaiming He, Xiangyu Zhang, Shaoqing Ren, and Jian Sun. Deep residual learning for image recognition. In Proceedings of the IEEE conference on computer vision and pattern recognition, pages 770-778, 2016. 1

[24] Zhezhi He and Deliang Fan. Simultaneously optimizing weight and quantizer of ternary neural network using truncated gaussian approximation. In Proceedings of the IEEE Conference on Computer Vision and Pattern Recognition, pages 11438-11446, 2019. 1

[25] Weizhe Hua, Yuan Zhou, Christopher De Sa, Zhiru Zhang, and G Edward Suh. Boosting the performance of cnn accelerators with dynamic fine-grained channel gating. In Proceedings of the 52nd Annual IEEE/ACM International Symposium on Microarchitecture, pages 139-150, 2019. 3 
[26] Weizhe Hua, Yuan Zhou, Christopher M De Sa, Zhiru Zhang, and G Edward Suh. Channel gating neural networks. In Advances in Neural Information Processing Systems, pages 1886-1896, 2019. 3

[27] Itay Hubara, Matthieu Courbariaux, Daniel Soudry, Ran El-Yaniv, and Yoshua Bengio. Quantized neural networks: Training neural networks with low precision weights and activations. The Journal of Machine Learning Research, 18(1):6869-6898, 2017. 1

[28] Masumi Ishikawa. Structural learning with forgetting. Neural networks, 9(3):509-521, 1996. 3

[29] Eric Jang, Shixiang $\mathrm{Gu}$, and Ben Poole. Categorical reparameterization with gumbel-softmax. arXiv preprint arXiv:1611.01144, 2016. 20

[30] Kaiyi Ji, Junjie Yang, and Yingbin Liang. Multi-step modelagnostic meta-learning: Convergence and improved algorithms. arXiv preprint arXiv:2002.07836, 2020. 2

[31] Yihan Jiang, Jakub Konečnỳ, Keith Rush, and Sreeram Kannan. Improving federated learning personalization via model agnostic meta learning. arXiv preprint arXiv:1909.12488, 2019. 2

[32] Peter Kairouz, H Brendan McMahan, Brendan Avent, Aurélien Bellet, Mehdi Bennis, Arjun Nitin Bhagoji, Keith Bonawitz, Zachary Charles, Graham Cormode, Rachel Cummings, et al. Advances and open problems in federated learning. arXiv preprint arXiv:1912.04977, 2019. 2

[33] Ehud D Karnin. A simple procedure for pruning backpropagation trained neural networks. IEEE transactions on neural networks, 1(2):239-242, 1990. 3

[34] Alex Krizhevsky, Geoffrey Hinton, et al. Learning multiple layers of features from tiny images. 2009. 7, 18

[35] Ilja Kuzborskij and Francesco Orabona. Fast rates by transferring from auxiliary hypotheses. Machine Learning, 106(2):171-195, 2017. 4

[36] Yann LeCun, Yoshua Bengio, and Geoffrey Hinton. Deep learning. nature, 521(7553):436-444, 2015. 1

[37] Yann LeCun, Léon Bottou, Yoshua Bengio, and Patrick Haffner. Gradient-based learning applied to document recognition. Proceedings of the IEEE, 86(11):2278-2324, 1998. 7

[38] Namhoon Lee, Thalaiyasingam Ajanthan, and Philip HS Torr. Snip: Single-shot network pruning based on connection sensitivity. arXiv preprint arXiv:1810.02340, 2018. 2, 3, 8

[39] Hao Li, Asim Kadav, Igor Durdanovic, Hanan Samet, and Hans Peter Graf. Pruning filters for efficient convnets. arXiv preprint arXiv:1608.08710, 2016. 3

[40] Zhenguo Li, Fengwei Zhou, Fei Chen, and Hang Li. Metasgd: Learning to learn quickly for few-shot learning. arXiv preprint arXiv:1707.09835, 2017. 2

[41] Sen Lin, Guang Yang, and Junshan Zhang. A collaborative learning framework via federated meta-learning. arXiv preprint arXiv:2001.03229, 2020. 3

[42] Sen Lin, Zhi Zhou, Zhaofeng Zhang, Xu Chen, and Junshan Zhang. Edge intelligence in the making: Optimization, deep learning, and applications. Synthesis Lectures on Learning, Networks, and Algorithms, 1(2):1-233, 2020. 1
[43] Tsung-Yi Lin, Priya Goyal, Ross Girshick, Kaiming He, and Piotr Dollár. Focal loss for dense object detection. In Proceedings of the IEEE international conference on computer vision, pages 2980-2988, 2017. 1

[44] Zhuang Liu, Jianguo Li, Zhiqiang Shen, Gao Huang, Shoumeng Yan, and Changshui Zhang. Learning efficient convolutional networks through network slimming. In Proceedings of the IEEE International Conference on Computer Vision, pages 2736-2744, 2017. 3

[45] Arun Mallya, Dillon Davis, and Svetlana Lazebnik. Piggyback: Adapting a single network to multiple tasks by learning to mask weights. In Proceedings of the European Conference on Computer Vision (ECCV), pages 67-82, 2018. 2

[46] Brendan McMahan, Eider Moore, Daniel Ramage, Seth Hampson, and Blaise Aguera y Arcas. Communicationefficient learning of deep networks from decentralized data. In Artificial Intelligence and Statistics, pages 1273-1282. PMLR, 2017. 2, 7

[47] Yurii Nesterov. Introductory lectures on convex optimization: A basic course, volume 87. Springer Science \& Business Media, 2013. 2, 5

[48] Alex Nichol, Joshua Achiam, and John Schulman. On first-order meta-learning algorithms. arXiv preprint arXiv:1803.02999, 2018. 2, 5

[49] Neal Parikh and Stephen Boyd. Proximal algorithms. Foundations and Trends in optimization, 1(3):127-239, 2014. 5, 6

[50] Vivek Ramanujan, Mitchell Wortsman, Aniruddha Kembhavi, Ali Farhadi, and Mohammad Rastegari. What's hidden in a randomly weighted neural network? In Proceedings of the IEEE/CVF Conference on Computer Vision and Pattern Recognition, pages 11893-11902, 2020. 2, 3

[51] Waseem Rawat and Zenghui Wang. Deep convolutional neural networks for image classification: A comprehensive review. Neural computation, 29(9):2352-2449, 2017. 1

[52] Russell Reed. Pruning algorithms-a survey. IEEE transactions on Neural Networks, 4(5):740-747, 1993. 3

[53] Felix Sattler, Simon Wiedemann, Klaus-Robert Müller, and Wojciech Samek. Robust and communication-efficient federated learning from non-iid data. IEEE transactions on neural networks and learning systems, 2019. 2

[54] Olivier Sigaud, Clément Masson, David Filliat, and Freek Stulp. Gated networks: an inventory. arXiv preprint arXiv:1512.03201, 2015. 3

[55] Virginia Smith, Chao-Kai Chiang, Maziar Sanjabi, and Ameet S Talwalkar. Federated multi-task learning. In $A d$ vances in Neural Information Processing Systems, pages 4424-4434, 2017. 2

[56] Minyoung Song, Jaehong Yoon, Eunho Yang, and Sung Ju Hwang. Rapid structural pruning of neural networks with set-based task-adaptive meta-pruning. arXiv preprint arXiv:2006.12139, 2020. 3

[57] Christian Szegedy, Alexander Toshev, and Dumitru Erhan. Deep neural networks for object detection. In Advances in neural information processing systems, pages 2553-2561, 2013. 1 
[58] Canh T Dinh, Nguyen Tran, and Tuan Dung Nguyen. Personalized federated learning with moreau envelopes. Advances in Neural Information Processing Systems, 33, 2020. 5, 7

[59] Xuezhi Wang and Jeff Schneider. Flexible transfer learning under support and model shift. In Advances in Neural Information Processing Systems, pages 1898-1906, 2014. 4

[60] Xin Wang, Fisher Yu, Zi-Yi Dou, Trevor Darrell, and Joseph E Gonzalez. Skipnet: Learning dynamic routing in convolutional networks. In Proceedings of the European Conference on Computer Vision (ECCV), pages 409-424, 2018. 1

[61] Andreas S Weigend, David E Rumelhart, and Bernardo A Huberman. Generalization by weight-elimination with application to forecasting. In Advances in neural information processing systems, pages 875-882, 1991. 3

[62] Wei Wen, Chunpeng Wu, Yandan Wang, Yiran Chen, and Hai Li. Learning structured sparsity in deep neural networks. In Advances in neural information processing systems, pages 2074-2082, 2016. 1, 2, 3

[63] Li Yang, Zhezhi He, Cao Yu, and Fan Deliang. Non-uniform dnn structured subnets sampling for dynamic inference. In 57th Design Automation Conference, 2020. 1

[64] Ming Yuan and Yi Lin. Model selection and estimation in regression with grouped variables. Journal of the Royal Statistical Society: Series B (Statistical Methodology), 68(1):49-67, 2006. 4

[65] Pan Zhou, Xiaotong Yuan, Huan Xu, Shuicheng Yan, and Jiashi Feng. Efficient meta learning via minibatch proximal update. In Advances in Neural Information Processing Systems, pages 1534-1544, 2019. 2, 4, 5, 6, 7, 12 


\section{Appendix}

\section{A. Auxiliary Lemmas}

To prove Theorem 1, we first restate some known results to be used later.

Lemma 4. [65] Assume that $g(u)$ is $\lambda$-strongly convex. Then we have

$$
\left\langle\nabla g(u), u-u^{*}\right\rangle \geq \lambda\left\|u-u^{*}\right\|^{2}
$$

where $u^{*}=\arg \min g(u)$.

Lemma 5. [19] For a sequence of learning rates $\left\{\alpha_{t}\right\}$ and the sequence $\left\{\gamma_{t}\right\}$ satisfies

$$
\gamma_{t} \leq\left(1-\alpha_{t}\right) \gamma_{t-1}+\tau_{t}, t=1,2, \ldots
$$

we have that $\gamma_{t} \leq \Gamma_{t} \sum_{i=1}^{t}\left(\tau_{i} / \Gamma_{i}\right)$ for any $t \geq 1$ where

$$
\Gamma_{t}= \begin{cases}1, & t=1 \\ \left(1-\alpha_{t}\right) \Gamma_{t-1}, & t \geq 2 .\end{cases}
$$

The next lemma characterizes the solution of the proximal updates in step 9 of Algorithm 1.

Lemma 6. [18] Let $h: X \rightarrow \mathbb{R}$ be a differentiable convex function and $V(x, z)$ be defined as

$$
V(x, z)=h(z)-[h(x)+\langle\nabla h(x), z-x\rangle] .
$$

For a given convex function $p: X \rightarrow \mathbb{R}$, the points $\tilde{x}, \tilde{y} \in X$ and the scalars $\mu_{1}, \mu_{2} \geq 0$, if

$$
u^{*} \in \arg \min \left\{p(u)+\mu_{1} V(\tilde{x}, u)+\mu_{2} V(\tilde{y}, u): u \in X\right\} \text {, }
$$

then for any $u \in X$, we have

$$
p\left(u^{*}\right)+\mu_{1} V\left(\tilde{x}, u^{*}\right)+\mu_{2} V\left(\tilde{y}, u^{*}\right) \leq p(u)+\mu_{1} V(\tilde{x}, u)+\mu_{2} V(\tilde{y}, u)-\left(\mu_{1}+\mu_{2}\right) V\left(u^{*}, u\right) .
$$

To show the convergence of the algorithm, we next need to connect the proximal update, based on the surrogate gradient $\nabla_{w t}$, with the true gradient $\nabla_{w} G\left(w_{t}^{p r}\right)$.

Lemma 7. The following inequalities hold for any $w$ :

$$
\begin{aligned}
\left\langle\nabla_{w} G\left(w_{t}^{p r}\right), w_{t}-w\right\rangle \leq & H(w)-H\left(w_{t}\right)+\left(\frac{1}{2 \eta_{t}}-\frac{1}{2}\right)\left[\left\|w_{t-1}-w\right\|^{2}-\left\|w_{t}-w\right\|^{2}\right] \\
& -\frac{1}{2 \eta_{t}}\left\|w_{t}-w_{t-1}\right\|^{2}+\frac{1}{2}\left\|\delta_{t}\right\|^{2}, \\
\left\langle\nabla_{w} G\left(w_{t}^{p r}\right), w_{t}^{a g}-w\right\rangle \leq & H(w)-H\left(w_{t}^{a g}\right)+\frac{1}{2 \beta_{t}}\left[\left\|w_{t}^{p r}-w\right\|^{2}-\left\|w_{t}^{a g}-w_{t}^{p r}\right\|^{2}\right]+\frac{1}{2}\left\|\delta_{t}\right\|^{2} .
\end{aligned}
$$

\section{B. Proof of Lemma 3}

Lemma 3. Suppose that Assumption 1 holds. For $\lambda>\rho$, the following equality holds:

$$
\nabla_{w, t}=\nabla_{w} G\left(w_{t}^{p r}\right)+\delta_{t},
$$

where $\left\|\delta_{t}\right\|^{2} \leq \frac{\lambda^{2} \xi_{t}}{(\lambda-\rho)^{2}}$.

Proof. Let $\tilde{w}_{t}^{i *}=\left(\tilde{\phi}_{t}^{i *}, \tilde{\theta}_{t}^{i *}\right)$ denote the optimal solution to the local optimization problem (3). We first investigate the structural property of the meta-learning objective function. Since $L_{i}$ is $\rho$-smooth, for $\lambda>\rho$, we can know that $G$ is $\frac{\lambda \rho}{\lambda+\rho}$-smooth based on Lemma 1. Then, based on the chain rule, we can know

$$
\begin{aligned}
\nabla_{w} G\left(w_{t}^{p r}\right) & =\frac{1}{N} \sum_{i=1}^{N}\left\{\left.\left(\frac{\partial \tilde{w}_{t}^{i *}}{\partial w}\right)^{T}\right|_{w=w_{t}^{p r}} \nabla_{w} L_{i}\left(\tilde{w}_{t}^{i *}\right)+\lambda\left(I-\left.\left(\frac{\partial \tilde{w}_{t}^{i *}}{\partial w}\right)^{T}\right|_{w=w_{t}^{p r}}\right)\left(w_{t}^{p r}-\tilde{w}_{t}^{i *}\right)\right\} \\
& =\frac{1}{N} \sum_{i=1}^{N}\left\{\lambda\left(w_{t}^{p r}-\tilde{w}_{t}^{i *}\right)+\left.\left(\frac{\partial \tilde{w}_{t}^{i *}}{\partial w}\right)^{T}\right|_{w=w_{t}^{p r}}\left[\nabla_{w} L_{i}\left(\tilde{w}_{t}^{i *}\right)-\lambda\left(w_{t}^{p r}-\tilde{w}_{t}^{i *}\right)\right]\right\} \\
& =\frac{\lambda}{N} \sum_{i=1}^{N}\left(w_{t}^{p r}-\tilde{w}_{t}^{i *}\right)
\end{aligned}
$$


where the last equality holds because the following first-order optimality condition for $\tilde{w}_{t}^{i *}$ :

$$
\nabla_{w} L_{i}\left(\tilde{w}_{t}^{i *}\right)+\lambda\left(\tilde{w}_{t}^{i *}-w_{t}^{p r}\right)=0 .
$$

Therefore, we can have that

$$
\begin{aligned}
\nabla_{w, t} & =\frac{\lambda}{N} \sum_{i=1}^{N}\left(w_{t}^{p r}-\tilde{w}_{t}^{i}\right) \\
& =\frac{\lambda}{N} \sum_{i=1}^{N}\left(w_{t}^{p r}-\tilde{w}_{t}^{i *}+\tilde{w}_{t}^{i *}-\tilde{w}_{t}^{i}\right) \\
& =\nabla_{w} G\left(w_{t}^{p r}\right)+\frac{\lambda}{N} \sum_{i=1}^{N}\left(\tilde{w}_{t}^{i *}-\tilde{w}_{t}^{i}\right) \\
& =\nabla_{w} G\left(w_{t}^{p r}\right)+\delta_{t} .
\end{aligned}
$$

Moreover, it is clear that $G_{i}$ is $(\lambda-\rho)$-strongly convex. Based on Lemma 4, it follows that

Hence, we can have

$$
\left\|\tilde{w}_{t}^{i *}-\tilde{w}_{t}^{i}\right\|^{2} \leq \frac{1}{(\lambda-\rho)^{2}}\left\|\nabla_{w} G_{i}\left(\tilde{w}_{t}^{i}\right)\right\|^{2} \leq \frac{\xi_{t}}{(\lambda-\rho)^{2}} .
$$

thereby completing the proof of Lemma 3.

$$
\begin{aligned}
\left\|\delta_{t}\right\|^{2} & =\lambda^{2}\left\|\frac{1}{N} \sum_{i=1}^{N}\left(\tilde{w}_{t}^{i *}-\tilde{w}_{t}^{i}\right)\right\|^{2} \\
& \leq \frac{\lambda^{2}}{N} \sum_{i=1}^{N}\left\|\tilde{w}_{t}^{i *}-\tilde{w}_{t}^{i}\right\|^{2} \\
& \leq \frac{\lambda^{2} \xi_{t}}{(\lambda-\rho)^{2}}
\end{aligned}
$$

\section{Proof of Lemma 7}

Lemma 7. The following inequalities hold for any $w$ :

$$
\begin{aligned}
\left\langle\nabla_{w} G\left(w_{t}^{p r}\right), w_{t}-w\right\rangle \leq & H(w)-H\left(w_{t}\right)+\left(\frac{1}{2 \eta_{t}}-\frac{1}{2}\right)\left[\left\|w_{t-1}-w\right\|^{2}-\left\|w_{t}-w\right\|^{2}\right] \\
& -\frac{1}{2 \eta_{t}}\left\|w_{t}-w_{t-1}\right\|^{2}+\frac{1}{2}\left\|\delta_{t}\right\|^{2}, \\
\left\langle\nabla_{w} G\left(w_{t}^{p r}\right), w_{t}^{a g}-w\right\rangle \leq & H(w)-H\left(w_{t}^{a g}\right)+\frac{1}{2 \beta_{t}}\left[\left\|w_{t}^{p r}-w\right\|^{2}-\left\|w_{t}^{a g}-w_{t}^{p r}\right\|^{2}\right]+\frac{1}{2}\left\|\delta_{t}\right\|^{2} .
\end{aligned}
$$

Proof. From the definition of the proximal update for the solution $w_{t}$, and Lemma 6, we can obtain that for any $w$

$$
\left\langle\nabla_{w, t}, w_{t}-w\right\rangle+H\left(w_{t}\right) \leq H(w)+\frac{1}{2 \eta_{t}}\left[\left\|w_{t-1}-w\right\|^{2}-\left\|w_{t}-w\right\|^{2}-\left\|w_{t}-w_{t-1}\right\|^{2}\right] .
$$

Based on Lemma 3, it follows that

$$
\begin{aligned}
& \left\langle\nabla_{w} G\left(w_{t}^{p r}\right)+\delta_{t}, w_{t}-w\right\rangle+H\left(w_{t}\right) \\
= & \left\langle\nabla_{w} G\left(w_{t}^{p r}\right), w_{t}-w\right\rangle+\left\langle\delta_{t}, w_{t}-w\right\rangle \\
\leq & H(w)+\frac{1}{2 \eta_{t}}\left[\left\|w_{t-1}-w\right\|^{2}-\left\|w_{t}-w\right\|^{2}-\left\|w_{t}-w_{t-1}\right\|^{2}\right]
\end{aligned}
$$


such that

$$
\begin{aligned}
& \left\langle\nabla_{w} G\left(w_{t}^{p r}\right), w_{t}-w\right\rangle \\
\leq & H(w)-H\left(w_{t}\right)+\frac{1}{2 \eta_{t}}\left[\left\|w_{t-1}-w\right\|^{2}-\left\|w_{t}-w\right\|^{2}-\left\|w_{t}-w_{t-1}\right\|^{2}\right]-\left\langle\delta_{t}, w_{t}-w\right\rangle \\
\leq & H(w)-H\left(w_{t}\right)+\frac{1}{2 \eta_{t}}\left[\left\|w_{t-1}-w\right\|^{2}-\left\|w_{t}-w\right\|^{2}-\left\|w_{t}-w_{t-1}\right\|^{2}\right]+\frac{1}{2}\left\|\delta_{t}\right\|^{2}+\frac{1}{2}\left\|w_{t}-w\right\|^{2} \\
\leq & H(w)-H\left(w_{t}\right)+\left(\frac{1}{2 \eta_{t}}-\frac{1}{2}\right)\left[\left\|w_{t-1}-w\right\|^{2}-\left\|w_{t}-w\right\|^{2}\right]-\frac{1}{2 \eta_{t}}\left\|w_{t}-w_{t-1}\right\|^{2}+\frac{1}{2}\left\|\delta_{t}\right\|^{2} .
\end{aligned}
$$

Similarly, for the solution $w_{t}^{a g}$, we have

$$
\left\langle\nabla_{w, t}, w_{t}^{a g}-w\right\rangle+H\left(w_{t}^{a g}\right) \leq H(w)+\frac{1}{2 \beta_{t}}\left[\left\|w_{t}^{p r}-w\right\|^{2}-\left\|w_{t}^{a g}-w\right\|^{2}-\left\|w_{t}^{a g}-w_{t}^{p r}\right\|^{2}\right] .
$$

Therefore, it follows that

$$
\begin{aligned}
& \left\langle\nabla_{w} G\left(w_{t}^{p r}\right), w_{t}^{a g}-w\right\rangle \\
\leq & H(w)-H\left(w_{t}^{a g}\right)+\frac{1}{2 \beta_{t}}\left[\left\|w_{t}^{p r}-w\right\|^{2}-\left\|w_{t}^{a g}-w\right\|^{2}-\left\|w_{t}^{a g}-w_{t}^{p r}\right\|^{2}\right]+\frac{1}{2}\left\|\delta_{t}\right\|^{2}+\frac{1}{2}\left\|w_{t}^{a g}-w\right\|^{2} \\
= & H(w)-H\left(w_{t}^{a g}\right)+\frac{1}{2 \beta_{t}}\left[\left\|w_{t}^{p r}-w\right\|^{2}-\left\|w_{t}^{a g}-w_{t}^{p r}\right\|^{2}\right]+\frac{1}{2}\left\|\delta_{t}\right\|^{2}-\left(\frac{1}{2 \beta_{t}}-\frac{1}{2}\right)\left\|w_{t}^{a g}-w\right\|^{2} \\
\leq & H(w)-H\left(w_{t}^{a g}\right)+\frac{1}{2 \beta_{t}}\left[\left\|w_{t}^{p r}-w\right\|^{2}-\left\|w_{t}^{a g}-w_{t}^{p r}\right\|^{2}\right]+\frac{1}{2}\left\|\delta_{t}\right\|^{2}
\end{aligned}
$$

for $\beta_{t} \leq 1$.

\section{Proof of Theorem 1}

Theorem 1. Suppose that Assumptions 1 and 2 hold. For any $t \geq 1$, let $\alpha_{t}=\frac{2}{t+1}, \beta_{t}<\frac{\lambda+\rho}{\lambda \rho}$ and $\eta_{t}$ satisfy:

$$
\alpha_{t} \eta_{t} \leq \beta_{t}, \frac{\alpha_{t}}{\Gamma_{t}}\left(\frac{1}{\eta_{t}}-1\right) \geq \frac{\alpha_{t+1}}{\Gamma_{t+1}}\left(\frac{1}{\eta_{t+1}}-1\right)
$$

Then, for $\lambda>\rho$, we have

$$
\begin{gathered}
\min _{t \in[1, T]}\left\|\mathcal{Q}\left(w_{t}^{p r}, \nabla_{w} G\left(w_{t}^{p r}\right), \beta_{t}\right)\right\|^{2} \leq \frac{24 \lambda^{3} \rho \sum_{t=1}^{T} \xi_{t}}{T(\lambda+\rho)(\lambda-\rho)^{2}} \\
+\frac{24(\lambda \rho)^{2}\left(\left\|w^{*}\right\|^{2}+2 M\right)}{T(\lambda+\rho)^{2}}+\frac{C\left\|w_{0}-w^{*}\right\|^{2}}{T^{2}(T+1)}
\end{gathered}
$$

where $C=\frac{24 \lambda \rho}{(\lambda+\rho)}\left(\frac{1}{\eta_{1}}-1\right)$

Proof. Based on the smoothness of $G$, we can conclude that

$$
\begin{gathered}
G\left(w_{t}^{a g}\right) \leq G\left(w_{t}^{p r}\right)+\left\langle\nabla_{w} G\left(w_{t}^{p r}\right), w_{t}^{a g}-w_{t}^{p r}\right\rangle+\frac{\lambda \rho}{2(\lambda+\rho)}\left\|w_{t}^{a g}-w_{t}^{p r}\right\|^{2}, \\
G\left(w_{t}^{p r}\right) \leq G(w)+\left\langle\nabla_{w} G\left(w_{t}^{p r}\right), w_{t}^{p r}-w\right\rangle+\frac{\lambda \rho}{2(\lambda+\rho)}\left\|w-w_{t}^{p r}\right\|^{2}, \\
G\left(w_{t}^{p r}\right) \leq G\left(w_{t-1}^{a g}\right)+\left\langle\nabla_{w} G\left(w_{t}^{p r}\right), w_{t}^{p r}-w_{t-1}^{a g}\right\rangle+\frac{\lambda \rho}{2(\lambda+\rho)}\left\|w_{t-1}^{a g}-w_{t}^{p r}\right\|^{2} .
\end{gathered}
$$


It follows that

$$
\begin{aligned}
& G\left(w_{t}^{p r}\right)-\left[\left(1-\alpha_{t}\right) G\left(w_{t-1}^{a g}\right)+\alpha_{t} G(w)\right] \\
= & \left(1-\alpha_{t}\right)\left[G\left(w_{t}^{p r}\right)-G\left(w_{t-1}^{a g}\right)\right]+\alpha_{t}\left[G\left(w_{t}^{p r}\right)-G(w)\right] \\
\leq & \left(1-\alpha_{t}\right)\left[\left\langle\nabla_{w} G\left(w_{t}^{p r}\right), w_{t}^{p r}-w_{t-1}^{a g}\right\rangle+\frac{\lambda \rho}{2(\lambda+\rho)}\left\|w_{t-1}^{a g}-w_{t}^{p r}\right\|^{2}\right] \\
& +\alpha_{t}\left[\left\langle\nabla_{w} G\left(w_{t}^{p r}\right), w_{t}^{p r}-w\right\rangle+\frac{\lambda \rho}{2(\lambda+\rho)}\left\|w-w_{t}^{p r}\right\|^{2}\right] \\
= & \left\langle\nabla_{w} G\left(w_{t}^{p r}\right), w_{t}^{p r}-\left[\left(1-\alpha_{t}\right) w_{t-1}^{a g}+\alpha_{t} w\right]\right\rangle+\frac{\lambda \rho\left(1-\alpha_{t}\right)}{2(\lambda+\rho)}\left\|w_{t-1}^{a g}-w_{t}^{p r}\right\|^{2}+\frac{\lambda \rho \alpha_{t}}{2(\lambda+\rho)}\left\|w-w_{t}^{p r}\right\|^{2} \\
= & \left\langle\nabla_{w} G\left(w_{t}^{p r}\right), w_{t}^{p r}-\left[\left(1-\alpha_{t}\right) w_{t-1}^{a g}+\alpha_{t} w\right]\right\rangle+\frac{\lambda \rho\left(1-\alpha_{t}\right) \alpha_{t}^{2}}{2(\lambda+\rho)}\left\|w_{t-1}^{a g}-w_{t-1}\right\|^{2}+\frac{\lambda \rho \alpha_{t}}{2(\lambda+\rho)}\left\|w-w_{t}^{p r}\right\|^{2}
\end{aligned}
$$

where the last equality is true because

$$
\begin{aligned}
\left\|w_{t-1}^{a g}-w_{t}^{p r}\right\| & =\left\|w_{t-1}^{a g}-\alpha_{t} w_{t-1}-\left(1-\alpha_{t}\right) w_{t-1}^{a g}\right\| \\
& =\alpha_{t}\left\|w_{t-1}^{a g}-w_{t-1}\right\| .
\end{aligned}
$$

Combining (11) and (14), we can have that

$$
\begin{aligned}
G\left(w_{t}^{a g}\right) \leq & \left(1-\alpha_{t}\right) G\left(w_{t-1}^{a g}\right)+\alpha_{t} G(w)+\left\langle\nabla_{w} G\left(w_{t}^{p r}\right), w_{t}^{a g}-w_{t}^{p r}\right\rangle+\frac{\lambda \rho}{2(\lambda+\rho)}\left\|w_{t}^{a g}-w_{t}^{p r}\right\|^{2} \\
& +\left\langle\nabla_{w} G\left(w_{t}^{p r}\right), w_{t}^{p r}-\left[\left(1-\alpha_{t}\right) w_{t-1}^{a g}+\alpha_{t} w\right]\right\rangle+\frac{\lambda \rho\left(1-\alpha_{t}\right) \alpha_{t}^{2}}{2(\lambda+\rho)}\left\|w_{t-1}^{a g}-w_{t-1}\right\|^{2}+\frac{\lambda \rho \alpha_{t}}{2(\lambda+\rho)}\left\|w-w_{t}^{p r}\right\|^{2} \\
= & \left(1-\alpha_{t}\right) G\left(w_{t-1}^{a g}\right)+\alpha_{t} G(w)+\left\langle\nabla_{w} G\left(w_{t}^{p r}\right), w_{t}^{a g}-\left[\left(1-\alpha_{t}\right) w_{t-1}^{a g}+\alpha_{t} w\right]\right\rangle \\
& +\frac{\lambda \rho}{2(\lambda+\rho)}\left\|w_{t}^{a g}-w_{t}^{p r}\right\|^{2}+\frac{\lambda \rho\left(1-\alpha_{t}\right) \alpha_{t}^{2}}{2(\lambda+\rho)}\left\|w_{t-1}^{a g}-w_{t-1}\right\|^{2}+\frac{\lambda \rho \alpha_{t}}{2(\lambda+\rho)}\left\|w-w_{t}^{p r}\right\|^{2} .
\end{aligned}
$$

Next, we need to bound the term $\left\langle\nabla_{w} G\left(w_{t}^{p r}\right), w_{t}^{a g}-\left[\left(1-\alpha_{t}\right) w_{t-1}^{a g}+\alpha_{t} w\right]\right\rangle$. Based on Lemma 7, it follows that

$\left\langle\nabla_{w} G\left(w_{t}^{p r}\right), w_{t}^{a g}-\left[\left(1-\alpha_{t}\right) w_{t-1}^{a g}+\alpha_{t} w\right]\right\rangle$

$$
\begin{aligned}
& =\left\langle\nabla_{w} G\left(w_{t}^{p r}\right), w_{t}^{a g}-\left(1-\alpha_{t}\right) w_{t-1}^{a g}-\alpha_{t} w_{t}\right\rangle+\alpha_{t}\left\langle\nabla_{w} G\left(w_{t}^{p r}\right), w_{t}-w\right\rangle \\
& \leq H\left(\left(1-\alpha_{t}\right) w_{t-1}^{a g}+\alpha_{t} w_{t}\right)-H\left(w_{t}^{a g}\right)+\frac{1}{2 \beta_{t}}\left[\left\|w_{t}^{p r}-\left(1-\alpha_{t}\right) w_{t-1}^{a g}-\alpha_{t} w_{t}\right\|^{2}-\left\|w_{t}^{a g}-w_{t}^{p r}\right\|^{2}\right]+\frac{1}{2}\left\|\delta_{t}\right\|^{2} \\
& \quad+\alpha_{t} H(w)-\alpha_{t} H\left(w_{t}\right)+\alpha_{t}\left(\frac{1}{2 \eta_{t}}-\frac{1}{2}\right)\left[\left\|w_{t-1}-w\right\|^{2}-\left\|w_{t}-w\right\|^{2}\right]-\frac{\alpha_{t}}{2 \eta_{t}}\left\|w_{t}-w_{t-1}\right\|^{2}+\frac{\alpha_{t}}{2}\left\|\delta_{t}\right\|^{2} \\
& \stackrel{(a)}{\leq}\left(1-\alpha_{t}\right) H\left(w_{t-1}^{a g}\right)+\alpha_{t} H\left(w_{t}\right)-H\left(w_{t}^{a g}\right)+\frac{1}{2 \beta_{t}}\left[\left\|w_{t}^{p r}-\left(1-\alpha_{t}\right) w_{t-1}^{a g}-\alpha_{t} w_{t}\right\|^{2}-\left\|w_{t}^{a g}-w_{t}^{p r}\right\|^{2}\right]+\frac{1}{2}\left\|\delta_{t}\right\|^{2}
\end{aligned}
$$$$
+\alpha_{t} H(w)-\alpha_{t} H\left(w_{t}\right)+\alpha_{t}\left(\frac{1}{2 \eta_{t}}-\frac{1}{2}\right)\left[\left\|w_{t-1}-w\right\|^{2}-\left\|w_{t}-w\right\|^{2}\right]-\frac{\alpha_{t}}{2 \eta_{t}}\left\|w_{t}-w_{t-1}\right\|^{2}+\frac{\alpha_{t}}{2}\left\|\delta_{t}\right\|^{2}
$$$$
\stackrel{(b)}{\leq}\left(1-\alpha_{t}\right) H\left(w_{t-1}^{a g}\right)+\alpha_{t} H(w)-H\left(w_{t}^{a g}\right)+\frac{1}{2 \beta_{t}}\left[\alpha_{t}^{2}\left\|w_{t}-w_{t-1}\right\|^{2}-\left\|w_{t}^{a g}-w_{t}^{p r}\right\|^{2}\right]+\frac{1+\alpha_{t}}{2}\left\|\delta_{t}\right\|^{2}
$$$$
+\alpha_{t}\left(\frac{1}{2 \eta_{t}}-\frac{1}{2}\right)\left[\left\|w_{t-1}-w\right\|^{2}-\left\|w_{t}-w\right\|^{2}\right]-\frac{\alpha_{t}}{2 \eta_{t}}\left\|w_{t}-w_{t-1}\right\|^{2}
$$$$
=\left(1-\alpha_{t}\right) H\left(w_{t-1}^{a g}\right)+\alpha_{t} H(w)-H\left(w_{t}^{a g}\right)+\alpha_{t}\left(\frac{1}{2 \eta_{t}}-\frac{1}{2}\right)\left[\left\|w_{t-1}-w\right\|^{2}-\left\|w_{t}-w\right\|^{2}\right]
$$$$
+\left(\frac{\alpha_{t}^{2}}{2 \beta_{t}}-\frac{\alpha_{t}}{2 \eta_{t}}\right)\left\|w_{t}-w_{t-1}\right\|^{2}+\frac{1+\alpha_{t}}{2}\left\|\delta_{t}\right\|^{2}-\frac{1}{2 \beta_{t}}\left\|w_{t}^{a g}-w_{t}^{p r}\right\|^{2}
$$$$
\stackrel{(c)}{\leq}\left(1-\alpha_{t}\right) H\left(w_{t-1}^{a g}\right)+\alpha_{t} H(w)-H\left(w_{t}^{a g}\right)+\alpha_{t}\left(\frac{1}{2 \eta_{t}}-\frac{1}{2}\right)\left[\left\|w_{t-1}-w\right\|^{2}-\left\|w_{t}-w\right\|^{2}\right]
$$$$
+\frac{1+\alpha_{t}}{2}\left\|\delta_{t}\right\|^{2}-\frac{1}{2 \beta_{t}}\left\|w_{t}^{a g}-w_{t}^{p r}\right\|^{2}
$$

where (a) holds because of the convexity of $H$, (b) is true because $w_{t}^{p r}=\left(1-\alpha_{t}\right) w_{t-1}^{a g}+\alpha_{t} w_{t-1}$, and (c) is true because 
$\alpha_{t} \eta_{t} \leq \beta_{t}$.

Continuing with (15), we can have that

$$
\begin{aligned}
G\left(w_{t}^{a g}\right) \leq & \left(1-\alpha_{t}\right) G\left(w_{t-1}^{a g}\right)+\alpha_{t} G(w)+\left(1-\alpha_{t}\right) H\left(w_{t-1}^{a g}\right)+\alpha_{t} H(w)-H\left(w_{t}^{a g}\right) \\
& +\frac{\lambda \rho}{2(\lambda+\rho)}\left\|w_{t}^{a g}-w_{t}^{p r}\right\|^{2}+\frac{\lambda \rho\left(1-\alpha_{t}\right) \alpha_{t}^{2}}{2(\lambda+\rho)}\left\|w_{t-1}^{a g}-w_{t-1}\right\|^{2}+\frac{\lambda \rho \alpha_{t}}{2(\lambda+\rho)}\left\|w-w_{t}^{p r}\right\|^{2} \\
& +\alpha_{t}\left(\frac{1}{2 \eta_{t}}-\frac{1}{2}\right)\left[\left\|w_{t-1}-w\right\|^{2}-\left\|w_{t}-w\right\|^{2}\right]+\frac{1+\alpha_{t}}{2}\left\|\delta_{t}\right\|^{2}-\frac{1}{2 \beta_{t}}\left\|w_{t}^{a g}-w_{t}^{p r}\right\|^{2} \\
= & \left(1-\alpha_{t}\right) G\left(w_{t-1}^{a g}\right)+\alpha_{t} G(w)+\left(1-\alpha_{t}\right) H\left(w_{t-1}^{a g}\right)+\alpha_{t} H(w)-H\left(w_{t}^{a g}\right) \\
& +\left(\frac{\lambda \rho}{2(\lambda+\rho)}-\frac{1}{2 \beta_{t}}\right)\left\|w_{t}^{a g}-w_{t}^{p r}\right\|^{2}+\frac{\lambda \rho\left(1-\alpha_{t}\right) \alpha_{t}^{2}}{2(\lambda+\rho)}\left\|w_{t-1}^{a g}-w_{t-1}\right\|^{2}+\frac{\lambda \rho \alpha_{t}}{2(\lambda+\rho)}\left\|w-w_{t}^{p r}\right\|^{2} \\
& +\alpha_{t}\left(\frac{1}{2 \eta_{t}}-\frac{1}{2}\right)\left[\left\|w_{t-1}-w\right\|^{2}-\left\|w_{t}-w\right\|^{2}\right]+\frac{1+\alpha_{t}}{2}\left\|\delta_{t}\right\|^{2}
\end{aligned}
$$

which indicates that

$$
\begin{aligned}
F\left(w_{t}^{a g}\right) \leq & \left(1-\alpha_{t}\right) F\left(w_{t-1}^{a g}\right)+\alpha_{t} F(w)+\left(\frac{\lambda \rho}{2(\lambda+\rho)}-\frac{1}{2 \beta_{t}}\right)\left\|w_{t}^{a g}-w_{t}^{p r}\right\|^{2}+\frac{\lambda \rho\left(1-\alpha_{t}\right) \alpha_{t}^{2}}{2(\lambda+\rho)}\left\|w_{t-1}^{a g}-w_{t-1}\right\|^{2} \\
& +\frac{\lambda \rho \alpha_{t}}{2(\lambda+\rho)}\left\|w-w_{t}^{p r}\right\|^{2}+\alpha_{t}\left(\frac{1}{2 \eta_{t}}-\frac{1}{2}\right)\left[\left\|w_{t-1}-w\right\|^{2}-\left\|w_{t}-w\right\|^{2}\right]+\frac{1+\alpha_{t}}{2}\left\|\delta_{t}\right\|^{2} .
\end{aligned}
$$

Based on Lemma 5, it follows that

$$
\begin{aligned}
& \frac{F\left(w_{T}^{a g}\right)-F(w)}{\Gamma_{T}} \\
\leq & \sum_{t=1}^{T}\left\{\frac{1}{\Gamma_{t}}\left(\frac{\lambda \rho}{2(\lambda+\rho)}-\frac{1}{2 \beta_{t}}\right)\left\|w_{t}^{a g}-w_{t}^{p r}\right\|^{2}+\frac{\lambda \rho\left(1-\alpha_{t}\right) \alpha_{t}^{2}}{2 \Gamma_{t}(\lambda+\rho)}\left\|w_{t-1}^{a g}-w_{t-1}\right\|^{2}\right\} \\
& +\sum_{t=1}^{T}\left\{\frac{\lambda \rho \alpha_{t}}{2 \Gamma_{t}(\lambda+\rho)}\left\|w-w_{t}^{p r}\right\|^{2}+\frac{\alpha_{t}}{\Gamma_{t}}\left(\frac{1}{2 \eta_{t}}-\frac{1}{2}\right)\left[\left\|w_{t-1}-w\right\|^{2}-\left\|w_{t}-w\right\|^{2}\right]+\frac{1+\alpha_{t}}{2 \Gamma_{t}}\left\|\delta_{t}\right\|^{2}\right\} \\
\leq & \sum_{t=1}^{T}\left\{\frac{1}{\Gamma_{t}}\left(\frac{\lambda \rho}{2(\lambda+\rho)}-\frac{1}{2 \beta_{t}}\right)\left\|w_{t}^{a g}-w_{t}^{p r}\right\|^{2}+\frac{\lambda \rho\left(1-\alpha_{t}\right) \alpha_{t}^{2}}{2 \Gamma_{t}(\lambda+\rho)}\left\|w_{t-1}^{a g}-w_{t-1}\right\|^{2}\right\} \\
& +\sum_{t=1}^{T}\left\{\frac{\lambda \rho \alpha_{t}}{2 \Gamma_{t}(\lambda+\rho)}\left\|w-w_{t}^{p r}\right\|^{2}+\frac{1+\alpha_{t}}{2 \Gamma_{t}}\left\|\delta_{t}\right\|^{2}\right\}+\left(\frac{1}{2 \eta_{1}}-\frac{1}{2}\right)\left\|w_{0}-w\right\|^{2}
\end{aligned}
$$

where the last inequality is true because

$$
\begin{aligned}
& \sum_{t=1}^{T} \frac{\alpha_{t}}{\Gamma_{t}}\left(\frac{1}{2 \eta_{t}}-\frac{1}{2}\right)\left[\left\|w_{t-1}-w\right\|^{2}-\left\|w_{t}-w\right\|^{2}\right] \\
\leq & \frac{\alpha_{1}}{\Gamma_{1}}\left(\frac{1}{2 \eta_{1}}-\frac{1}{2}\right) \sum_{t=1}^{T}\left[\left\|w_{t-1}-w\right\|^{2}-\left\|w_{t}-w\right\|^{2}\right] \\
= & \left(\frac{1}{2 \eta_{1}}-\frac{1}{2}\right)\left[\left\|w_{0}-w\right\|^{2}-\left\|w_{T}-w\right\|^{2}\right] \\
\leq & \left(\frac{1}{2 \eta_{1}}-\frac{1}{2}\right)\left\|w_{0}-w\right\|^{2}
\end{aligned}
$$

considering that $\frac{\alpha_{t}}{\Gamma_{t}}\left(\frac{1}{2 \eta_{t}}-\frac{1}{2}\right) \geq \frac{\alpha_{t+1}}{\Gamma_{t+1}}\left(\frac{1}{2 \eta_{t+1}}-\frac{1}{2}\right)$ for any $t \geq 1$. 
Therefore, by rearranging (16), we can have that for any $w$ the following holds

$$
\begin{aligned}
& \sum_{t=1}^{T} \frac{1}{\Gamma_{t}}\left(\frac{1}{2 \beta_{t}}-\frac{\lambda \rho}{2(\lambda+\rho)}\right)\left\|w_{t}^{a g}-w_{t}^{p r}\right\|^{2} \\
& \leq \frac{F(w)-F\left(w_{T}^{a g}\right)}{\Gamma_{T}}+\sum_{t=1}^{T} \frac{\lambda \rho \alpha_{t}}{2 \Gamma_{t}(\lambda+\rho)}\left[\left(1-\alpha_{t}\right) \alpha_{t}\left\|w_{t-1}^{a g}-w_{t-1}\right\|^{2}+\left\|w-w_{t}^{p r}\right\|^{2}\right] \\
& +\left(\frac{1}{2 \eta_{1}}-\frac{1}{2}\right)\left\|w_{0}-w\right\|^{2}+\sum_{t=1}^{T} \frac{1+\alpha_{t}}{2 \Gamma_{t}}\left\|\delta_{t}\right\|^{2} \\
& \leq \frac{F(w)-F\left(w_{T}^{a g}\right)}{\Gamma_{T}}+\sum_{t=1}^{T} \frac{\lambda \rho \alpha_{t}}{\Gamma_{t}(\lambda+\rho)}\left[\alpha_{t}\left(1-\alpha_{t}\right)\left(\left\|w_{t-1}^{a g}\right\|^{2}+\left\|w_{t-1}\right\|^{2}\right)+\|w\|^{2}+\left\|w_{t}^{p r}\right\|^{2}\right] \\
& +\left(\frac{1}{2 \eta_{1}}-\frac{1}{2}\right)\left\|w_{0}-w\right\|^{2}+\sum_{t=1}^{T} \frac{1+\alpha_{t}}{2 \Gamma_{t}}\left\|\delta_{t}\right\|^{2} \\
& \stackrel{(a)}{\leq} \frac{F(w)-F\left(w_{T}^{a g}\right)}{\Gamma_{T}}+\sum_{t=1}^{T} \frac{\lambda \rho \alpha_{t}}{\Gamma_{t}(\lambda+\rho)}\left[\alpha_{t}\left(1-\alpha_{t}\right)\left(\left\|w_{t-1}^{a g}\right\|^{2}+\left\|w_{t-1}\right\|^{2}\right)+\|w\|^{2}+\left(1-\alpha_{t}\right)\left\|w_{t-1}^{a g}\right\|^{2}+\alpha_{t}\left\|w_{t-1}\right\|^{2}\right] \\
& +\left(\frac{1}{2 \eta_{1}}-\frac{1}{2}\right)\left\|w_{0}-w\right\|^{2}+\sum_{t=1}^{T} \frac{1+\alpha_{t}}{2 \Gamma_{t}}\left\|\delta_{t}\right\|^{2} \\
& \leq \frac{F(w)-F\left(w_{T}^{a g}\right)}{\Gamma_{T}}+\sum_{t=1}^{T} \frac{\lambda \rho \alpha_{t}}{\Gamma_{t}(\lambda+\rho)}\left[\|w\|^{2}+\left\|w_{t-1}^{a g}\right\|^{2}+\left\|w_{t-1}\right\|^{2}\right]+\left(\frac{1}{2 \eta_{1}}-\frac{1}{2}\right)\left\|w_{0}-w\right\|^{2}+\sum_{t=1}^{T} \frac{1+\alpha_{t}}{2 \Gamma_{t}}\left\|\delta_{t}\right\|^{2} \\
& \stackrel{(b)}{\leq} \frac{F(w)-F\left(w_{T}^{a g}\right)}{\Gamma_{T}}+\sum_{t=1}^{T} \frac{\lambda \rho \alpha_{t}}{\Gamma_{t}(\lambda+\rho)}\left[\|w\|^{2}+2 M\right]+\left(\frac{1}{2 \eta_{1}}-\frac{1}{2}\right)\left\|w_{0}-w\right\|^{2}+\sum_{t=1}^{T} \frac{1+\alpha_{t}}{2 \Gamma_{t}}\left\|\delta_{t}\right\|^{2} \\
& \stackrel{(c)}{=} \frac{F(w)-F\left(w_{T}^{a g}\right)}{\Gamma_{T}}+\frac{\lambda \rho}{\Gamma_{T}(\lambda+\rho)}\left(\|w\|^{2}+2 M\right)+\left(\frac{1}{2 \eta_{1}}-\frac{1}{2}\right)\left\|w_{0}-w\right\|^{2}+\sum_{t=1}^{T} \frac{1+\alpha_{t}}{2 \Gamma_{t}}\left\|\delta_{t}\right\|^{2}
\end{aligned}
$$

where (a) is because of the convexity of $\|\cdot\|^{2}$ and $w_{t}^{p r}=\left(1-\alpha_{t}\right) w_{t-1}^{a g}+\alpha_{t} w_{t-1}$, (b) is because the solution to the proximal optimization problem is bound from above, and (c) holds because

$$
\sum_{t=1}^{T} \frac{\alpha_{t}}{\Gamma_{t}}=\frac{1}{\Gamma_{1}}+\sum_{t=2}^{T}\left(\frac{1}{\Gamma_{t}}-\frac{1}{\Gamma_{t-1}}\right)=\frac{1}{\Gamma_{T}} .
$$

Let $w^{*}$ be the optimal solution of $F(w)$, i.e., $F\left(w^{*}\right)=\min _{w} F(w)$. Since $\mathcal{Q}\left(w_{t}^{p r}, \nabla_{w} G\left(w_{t}^{p r}\right), \beta_{t}\right)=\frac{1}{\beta_{t}}\left(w_{t}^{p r}-w_{t}^{a g}\right)$, it is clear that

$$
\begin{aligned}
& \sum_{t=1}^{T} \frac{\beta_{t}^{2}}{\Gamma_{t}}\left(\frac{1}{2 \beta_{t}}-\frac{\lambda \rho}{2(\lambda+\rho)}\right)\left\|\mathcal{Q}\left(w_{t}^{p r}, \nabla_{w} G\left(w_{t}^{p r}\right), \beta_{t}\right)\right\|^{2} \\
\leq & \frac{F\left(w^{*}\right)-F\left(w_{T}^{a g}\right)}{\Gamma_{T}}+\frac{\lambda \rho}{\Gamma_{T}(\lambda+\rho)}\left(\left\|w^{*}\right\|^{2}+2 M\right)+\left(\frac{1}{2 \eta_{1}}-\frac{1}{2}\right)\left\|w_{0}-w^{*}\right\|^{2}+\sum_{t=1}^{T} \frac{1+\alpha_{t}}{2 \Gamma_{t}}\left\|\delta_{t}\right\|^{2} \\
\leq & \frac{\lambda \rho}{\Gamma_{T}(\lambda+\rho)}\left(\left\|w^{*}\right\|^{2}+2 M\right)+\left(\frac{1}{2 \eta_{1}}-\frac{1}{2}\right)\left\|w_{0}-w^{*}\right\|^{2}+\sum_{t=1}^{T} \frac{1+\alpha_{t}}{2 \Gamma_{t}}\left\|\delta_{t}\right\|^{2}
\end{aligned}
$$

which implies that

$$
\begin{aligned}
& \min _{t \in[1, T]}\left\|\mathcal{Q}\left(w_{t}^{p r}, \nabla_{w} G\left(w_{t}^{p r}\right), \beta_{t}\right)\right\|^{2} \\
\leq & {\left[\sum_{t=1}^{T} \frac{\beta_{t}^{2}}{\Gamma_{t}}\left(\frac{1}{2 \beta_{t}}-\frac{\lambda \rho}{2(\lambda+\rho)}\right)\right]^{-1}\left[\frac{\lambda \rho}{\Gamma_{T}(\lambda+\rho)}\left(\left\|w^{*}\right\|^{2}+2 M\right)+\left(\frac{1}{2 \eta_{1}}-\frac{1}{2}\right)\left\|w_{0}-w^{*}\right\|^{2}+\sum_{t=1}^{T} \frac{1+\alpha_{t}}{2 \Gamma_{t}}\left\|\delta_{t}\right\|^{2}\right] . }
\end{aligned}
$$




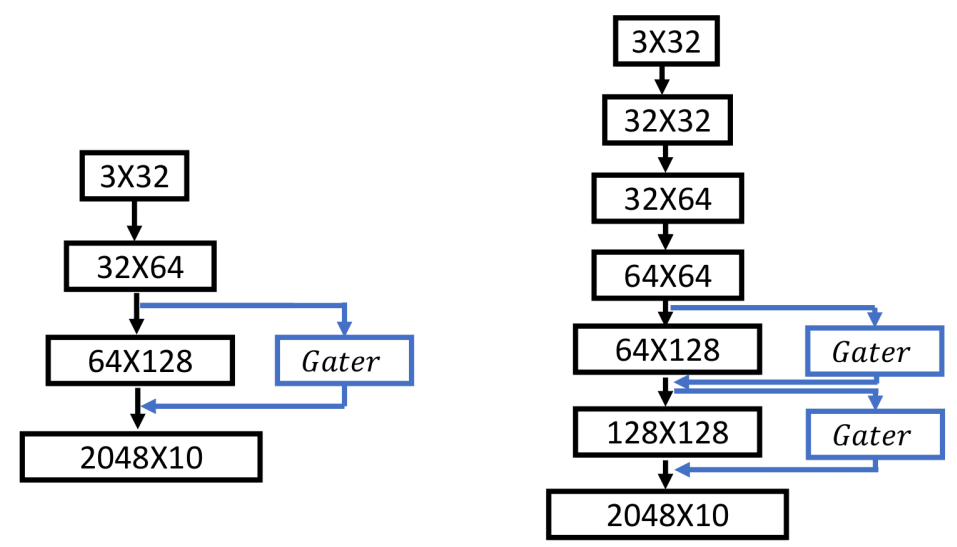

(a)

(b)

Figure 5. The DNN architectures used in the experiments. We use (a) for experiments in Table 1-2, and (b) for experiments in Table 3-6.

For convenience, choose $\alpha_{t}=\frac{2}{t+1}, \beta_{t}<\frac{\lambda+\rho}{\lambda \rho}$, and $\eta_{t}$ to satisfy that

$$
\alpha_{t} \eta_{t} \leq \beta_{t}, \frac{\alpha_{t}}{\Gamma_{t}}\left(\frac{1}{\eta_{t}}-1\right) \geq \frac{\alpha_{t+1}}{\Gamma_{t+1}}\left(\frac{1}{\eta_{t+1}}-1\right),
$$

for any $t \in[1, T]$. Then we can have

$$
\begin{aligned}
& \min _{t \in[1, T]}\left\|\mathcal{Q}\left(w_{t}^{p r}, \nabla_{w} G\left(w_{t}^{p r}\right), \beta_{t}\right)\right\|^{2} \\
\leq & \frac{48 \lambda \rho}{T^{2}(T+1)(\lambda+\rho)}\left[\frac{T(T+1) \lambda \rho}{2(\lambda+\rho)}\left(\left\|w^{*}\right\|^{2}+2 M\right)+\left(\frac{1}{2 \eta_{1}}-\frac{1}{2}\right)\left\|w_{0}-w^{*}\right\|^{2}+\frac{T(T+1)}{2} \sum_{t=1}^{T}\left\|\delta_{t}\right\|^{2}\right] \\
= & \frac{24(\lambda \rho)^{2}}{T(\lambda+\rho)^{2}}\left(\left\|w^{*}\right\|^{2}+2 M\right)+\frac{C}{T^{2}(T+1)}\left\|w_{0}-w^{*}\right\|^{2}+\frac{24 \lambda \rho}{T(\lambda+\rho)} \sum_{t=1}^{T}\left\|\delta_{t}\right\|^{2} \\
\leq & \frac{24(\lambda \rho)^{2}}{T(\lambda+\rho)^{2}}\left(\left\|w^{*}\right\|^{2}+2 M\right)+\frac{C}{T^{2}(T+1)}\left\|w_{0}-w^{*}\right\|^{2}+\frac{24 \lambda^{3} \rho}{T(\lambda+\rho)(\lambda-\rho)^{2}} \sum_{t=1}^{T} \xi_{t} .
\end{aligned}
$$

\section{E. More Experimental Results}

\section{E.1. Experimental setup}

To obtain a comprehensive understanding of the performance of MetaGater, we conduct more experiments of image classification on CIFAR-10 and CIFAR-100 [34] by using a DNN with more complex structure. Specifically, as shown in Fig. 5, we consider a seven-layer convolutional neural network with six convolutional layers, followed by a fully connected layer. Each convolution layer is followed by a ReLU activation layer. Besides, we also adopt two max pooling layers and one average pooling layer to shrink the feature map dimension. And we integrate a channel gating module with the fifth and sixth convolutional layers, respectively.

It is worth noting that we integrate the channel gating module only at the layers near the output layer, aiming to 1) minimize the introduced model overhead, and 2) preserve the more important features in the layers near the input layer. In fact, we have also tried to integrate the channel gating module only at the second convolutional layer of the model (a) shown in Fig. 5, and the testing accuracy degrades compared with the the marginal accuracy drop in Table 2. Such a performance degradation makes sense as the low-level features captured by the first few layers have more critical impact on the overall performance, in contrast to the high-level features in the last few layers. Besides, we study the cross-entropy loss and use Group Lasso to prompt the sparsity of the channel gating module.

Since a resource-limited node often has only a small local dataset for learning, we further investigate the performance of MetaGater under different sizes of local datasets. In particular, we consider two different regimes of the local dataset size:

- Moderate local dataset size. For CIFAR-10, we distribute the dataset among $N=20$ nodes, where each node only has 


\begin{tabular}{c|cccc}
\hline Dataset & Method & Local update & Accuracy(\%) & Training time(s) \\
\hline \multirow{4}{*}{ CIFAR-10 } & FedAvg & 1 & $74.2 \pm 1.2$ & 1677 \\
\cline { 2 - 5 } & Per-FedAvg & 1 & $76.8 \pm 1.8$ & 3098 \\
\cline { 2 - 5 } & MetaGater w/o gating & 1 & $\mathbf{8 5 . 3} \pm \mathbf{2 . 1}$ & 1939 \\
\cline { 2 - 5 } & MetaGater w/ gating & 1 & $\mathbf{8 5 . 1} \pm \mathbf{3 . 6}$ & 2558 \\
\hline
\end{tabular}

Table 3. Accuracy comparison for MetaGater, Per-FedAvg, FedAvg on CIFAR-10 with moderate local datasets. Clearly, MetaGater achieves the best accuracy among all methods.

\begin{tabular}{c|cccc}
\hline Dataset & Method & Accuracy(\%) & Sparsity(\%) & Learning time(s) \\
\hline \multirow{3}{*}{ CIFAR-10 } & MetaGater w/o gating & $\mathbf{8 5 . 3} \pm \mathbf{2 . 1}$ & 0 & 1.6 \\
\cline { 2 - 5 } & MetaSNIP & $83.4 \pm 3.4$ & $23 \pm 2.4$ & 1.5 \\
\cline { 2 - 5 } & MetaGater w/ gating & $\mathbf{8 5 . 1} \pm \mathbf{3 . 6}$ & $23 \pm 3.7$ & 1.4 \\
\hline
\end{tabular}

Table 4. Fast adaptation performance comparison on CIFAR-10 with moderate local datasets. Compared with MetaSNIP, MetaGater has a better accuracy and a larger sparsity range.

the data samples from two classes and the number of samples per node is in the range of [221,2792]. We randomly select 10 nodes for each round in federated meta-learning, and 10 target nodes for fast adaptation.

- Small local dataset size. For both CIFAR-10 and CIFAR-100, we distribute the dataset among $N=20$ nodes, where the number of samples per node is in the range of $[30,100]$. We randomly select 10 nodes for each round in federated meta-learning, and 10 target nodes for fast adaptation. The difference is that each node has the data samples from two classes in CIFAR-10, but five classes in CIFAR-100.

We evaluate the performance under a fixed number of communication rounds, i.e., $T=400$. For $t$-th round, the learning rate $\alpha_{t}=\frac{2}{t+1}$, and we choose $\beta_{t}=\alpha_{t} \eta_{t}=1$. Besides, $\lambda=0.2$. During federated meta-learning, we run gradient descent for one local update to solve the local minimization problem (5) for each training task. For fast adaptation at target nodes, we only run one-step gradient descent to fine-tune both meta-backbone network and meta-gating module. We evaluate the testing accuracy at the target nodes, and repeat all the experiments for 5 times to obtain the average performance.

\section{E.2. Performance of MetaGater with moderate local datasets}

We evaluate the performance of MetaGater with moderate local datasets on CIFAR-10. As shown in Table 3, MetaGater still achieves the best accuracy performance. It is also worth to mention that for MetaGater with gating, even though extra training time is needed to jointly train the meta-backbone network and the meta-gating module compared to training MetaGater without gating, it still takes the similar training time but achieves much higher accuracy, in contrast to Per-FedAvg. Moreover, it can be seen from Table 4 that with channel gating module, the target node is able to quickly obtain a more compact model for efficient inference with almost same accuracy performance, compared with MetaGater without gating module. And MetaGater clearly outperforms MetaSNIP in terms of the testing accuracy and demonstrates a larger diversity of the achieved model sparsity, in a similar speed with MetaSNIP.

\section{E.3. Performance of MetaGater with small local datasets}

We evaluate the performance of MetaGater with small local datasets on both CIFAR-10 and CIFAR-100. As illustrated in Table 5, MetaGater significantly outperforms other federated meta-learning methods, and the performance gap is even larger compared with the case under moderate local datasets. And Table 6 shows that MetaGater still performs better than MetaSNIP. More importantly, as the model becomes deeper and the local datasets becomes smaller, the accuracy gap between MetaGater w/ gating and MetaGater w/o gating decreases, and MetaGater w/ gating can even achieve a better accuracy compared with MetaGater w/o gating. This is because the backbone network will become relatively overparameterized, and the channel gating module can accurately select the task-specific subnet with the data-dependent important filters that lead to a better accuracy performance.

In a nutshell, under different model sizes and various local dataset sizes, MetaGater clearly achieves better accuracy compared with other federated meta-learning methods, and outperforms MetaSNIP, in the sense that a better accuracy and a larger sparsity diversity can be attained by MetaGater in a similar speed with MetaSNIP. Moreover, since the meta-gating 


\begin{tabular}{c|cccc}
\hline Dataset & Method & Local update & Accuracy $(\%)$ & Training time(s) \\
\hline \multirow{4}{*}{ CIFAR-10 } & FedAvg & 1 & $58.7 \pm 1.2$ & 187 \\
\cline { 2 - 5 } & Per-FedAvg & 1 & $60.5 \pm 1.9$ & 339 \\
\cline { 2 - 5 } & MetaGater w/o gating & 1 & $\mathbf{7 8 . 6} \pm \mathbf{2 . 3}$ & 189 \\
\cline { 2 - 5 } & MetaGater w/ gating & 1 & $\mathbf{7 8 . 4} \pm \mathbf{3 . 5}$ & 356 \\
\hline \multirow{4}{*}{ CIFAR-100 } & FedAvg & 1 & $42.8 \pm 1.1$ & 192 \\
\cline { 2 - 5 } & Per-FedAvg & 1 & $56.6 \pm 1.6$ & 367 \\
\cline { 2 - 5 } & MetaGater w/o gating & 1 & $\mathbf{6 8 . 4} \pm \mathbf{2 . 1}$ & 208 \\
\cline { 2 - 5 } & MetaGater w/ gating & 1 & $\mathbf{6 8 . 6} \pm \mathbf{4 . 5}$ & 399 \\
\hline
\end{tabular}

Table 5. Accuracy comparison for MetaGater, Per-FedAvg, FedAvg on CIFAR-10 and CIFAR-100 with small local datasets. Clearly, MetaGater achieves the best accuracy among all methods.

\begin{tabular}{c|cccc}
\hline Dataset & Method & Accuracy(\%) & Sparsity(\%) & Learning time(s) \\
\hline \multirow{3}{*}{ CIFAR-10 } & MetaGater w/o gating & $\mathbf{7 8 . 6} \pm \mathbf{2 . 3}$ & 0 & 0.56 \\
\cline { 2 - 5 } & MetaSNIP & $76.7 \pm 2.4$ & $17 \pm 1.3$ & 0.55 \\
\cline { 2 - 5 } & MetaGater w/ gating & $\mathbf{7 8 . 4} \pm \mathbf{3 . 5}$ & $17 \pm 2.1$ & 0.51 \\
\hline \multirow{3}{*}{ CIFAR-100 } & MetaGater w/o gating & $\mathbf{6 8 . 4} \pm \mathbf{2 . 1}$ & 0 & 0.61 \\
\cline { 2 - 5 } & MetaSNIP & $66.9 \pm 3.7$ & $21 \pm 2.3$ & 0.57 \\
\cline { 2 - 5 } & MetaGater w/ gating & $\mathbf{6 8 . 6} \pm \mathbf{4 . 5}$ & $21 \pm 3.6$ & 0.52 \\
\hline
\end{tabular}

Table 6. Fast adaptation performance comparison on CIFAR-10 and CIFAR-100 with small local datasets. Compared with MetaSNIP, MetaGater has a better accuracy and a larger sparsity range.

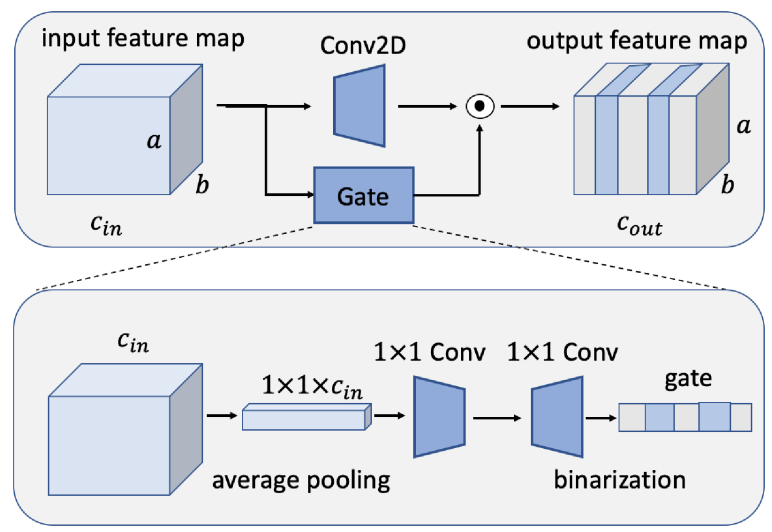

Figure 6. The channel gating module for a convolution layer.

module is able to effectively capture the important filters of a good meta-backbone network and hence sparsity structure across tasks, a task-specific conditional channel gated network for a new task can be quickly adapted from the meta-initialization with only a small local dataset. In this way, the obtained channel gated network with structural sparsity patterns significantly reduces the network size for computing while still guaranteeing the testing accuracy, by quickly selecting the important filters for the new task.

\section{F. Channel Gating Module}

Fig. 6 shows the channel gating module in detail. To generate a binary mask, a straightforward way is to use a binarization function, which utilizes a hard threshold to take binary on/off decision. However, such a discrete function is non-differential during back-propagation. A widely used solution is straight-through estimator [8] where the incoming gradient is equal to the outgoing gradient. To better estimate the gradient, we use Gumbel Softmax trick [29]. Specifically, we utilize the hard threshold during forward pass to generate binary masks and the differential softmax function during back-propagation. Note that, the temperature of the Gumbel Softmax is set to be 1 for all the experiments. 